\title{
Introduction
}

Measurements of the acceleration environment on the U.S. Space Shuttle have demonstrated that the on-orbit environment will exceed the requirements for micro-gravity experiments [1]. To meet the required level of microgravity isolation many space-science experiments will likely require some attenuation of the nominal ISS acceleration environment. The expected acceleration levels over a mid-range of frequencies above $0.01 \mathrm{~Hz}$ and below 10 Hz. are particularly high occasionally reaching milli-g levels [1]. Three orders-of-magnitude attenuation of the induced accelerations on the experiment platform, with frequency roll-off of $20 \mathrm{db} /$ decade over a range from $0.01 \mathrm{~Hz}$. to $10 \mathrm{~Hz}$, has been established as a design requirement for a vibration isolation system [2]. To meet this relatively stringent requirement it has been established that active vibration control is necessary [1].

The Glovebox Integrated Microgravity Isolation Technology (g-LIMIT) is designed to isolate experiments from the medium frequency $(>0.01 \mathrm{~Hz})$ vibrations on the ISS, while passing the quasi-static $(<0.01 \mathrm{~Hz})$ accelerations to the experiment [2]. The acceleration-attenuation capability of g-LIMIT is limited primarily by two factors: (1) the character of the umbilical required between the g-LIMIT base (stator) and the g-LIMIT experiment platform (flotor), and (2) the allowed stator-to-flotor rattlespace. A primary goal in g-LIMIT design was to isolate at the individual experiment, rather than entire rack level; ideally g-LIMIT isolates only the sensitive elements of an experiment. This typically results in a stator-to-flotor umbilical that can be greatly reduced in size and in the services it must provide. In the current design, g-LIMIT employs three umbilicals to provide experiments with power, and with data-acquisition and control services [3]. 
In order to design controllers for g-LIMIT it was necessary to develop an appropriate dynamic model of the system. The design methods employed in the present paper require a linearized system model in state-space form. A six-degree-of-freedom (6-DOF) state model, augmented with absolute acceleration states, was developed in a form appropriate for an optimal control design for g-LIMIT [1]. A set of representative parameters used in the state space model for controller design is provided in the following section.

\section{g-LIMIT State Space Model}

The linearized state-space equations of motion for g-LIMIT were used to develop linear optimal controller designs [1]. To construct the state space model a set of representative flotor and umbilical parameters, shown in Table 1, were used in the controller design study. There are three umbilicals included in this model of g-LIMIT. The translational and rotational stiffness matrices for each umbilical were assumed to be diagonal along an umbilical-fixed set of coordinate directions. These diagonal stiffness values are included in Table 2. Similarity transformations of these diagonal matrices were performed assuming a coordinate transformation from each local umbilical-fixed reference frame to the stator-fixed frame. First, a coordinate rotation about the stator-fixed $+Z$ axis of $120 \mathrm{deg}$ and $240 \mathrm{deg}$ was performed to align umbilcal \#2 and \#3, in their respective home locations. Then, for each umbilical, a 20 deg rotation about each coordinate axis was used to represent an arbitrary misalignment of the diagonal-stiffiness directions to the stator-fixed directions. The translational and rotational damping matrices were assumed to be proportional to the stiffness matrices with a damping ratio of $3 \%$ used for all of the vibrational modes. The resulting umbilical stiffness and damping matricies are given in reference [1]. They are not included in this paper but can easily be computed via coordinate transformations of the diagonal stiffness terms given in Table 2. All stiffness and damping translation/rotational 
cross-terms, i.e. $K_{t r}, K_{r t}, C_{t r}$, and $C_{r t}$, were considered to be zero. In addition to the parameters listed in Table \#1 the actuator currents were set to initial bias values. These bias currents were required to produce a bias force and moment to move the flotor from its assumed relaxed position to the home location. The flotor relaxed-position was assumed to be $2 \mathrm{~mm}$ from the homeposition and misaligned by approximately 2 deg. about each stator-fixed coordinate axis. This resulted in the following set of bias current values; $I_{B_{1}}=-0.264 \mathrm{~A}, I_{B_{4}}=-0.159 \mathrm{~A}$, and $I_{l,}=0.123 \mathrm{~A}$. 
Table 1 - g-LIMIT Parameters

\begin{tabular}{|c|c|c|}
\hline Palrameter & Symbol & Value \\
\hline Flotor Mass & $m$ & $15.12 \mathrm{~kg}$ \\
\hline Flotor Moments of Inertia & $\begin{array}{l}I_{x x} \\
I_{y y} \\
I_{z z}\end{array}$ & $\begin{array}{l}0.50 \mathrm{~kg} \mathrm{~m}^{2} \\
0.62 \mathrm{~kg} \mathrm{~m}^{2} \\
0.18 \mathrm{~kg} \mathrm{~m}^{2}\end{array}$ \\
\hline Flotor Products of Inertia & $\begin{array}{l}I_{x v} \\
I_{t=} \\
I_{r:}\end{array}$ & $\begin{array}{l}\mathrm{le}-4 \mathrm{~kg} \mathrm{~m}^{2} \\
-1 \mathrm{e}-4 \mathrm{~kg} \mathrm{~m}^{2} \\
-8 \mathrm{e}-4 \mathrm{~kg} \mathrm{~m}^{2}\end{array}$ \\
\hline $\begin{array}{l}\text { Umbilical Locations (F) } \\
\text { (3 Umbilicals) }\end{array}$ & ${ }^{(r)} r_{F} r_{n}$ & 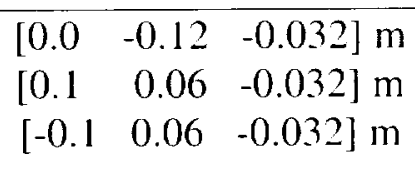 \\
\hline $\begin{array}{l}\text { Actuator Current Vectors (S) } \\
\text { (6 Actuator Coils) }\end{array}$ & (5) $\hat{\underline{I}}_{i}$ & 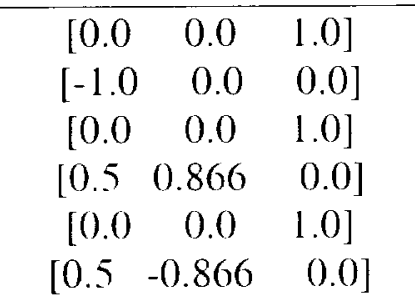 \\
\hline $\begin{array}{l}\text { Actuator Magnet B-Field Vectors } \\
\text { (F) } \\
\text { (3 Actuator Magnets) }\end{array}$ & ${ }^{(1)} \hat{\underline{B}}_{i}$ & $\begin{array}{lll}{[0.0} & 1.0 & 0.0 \\
{[0.0} & 1.0 & 0.0] \\
{[0.866} & -0.5 & 0.0] \\
{[0.866} & -0.5 & 0.0] \\
{[-0.866} & -0.5 & 0.0] \\
{[-0.866} & -0.5 & 0.0]\end{array}$ \\
\hline Actuator Constant & $\left(L_{i} B_{i}\right)$ & $1.0 \mathrm{~N} / \mathrm{Amp}$ \\
\hline
\end{tabular}

Table 2 - Diagonal Stiffness Parameters

\begin{tabular}{|l|c|c|}
\hline & Translational & Rotational \\
\hline & {$[\mathrm{N} / \mathrm{m}]$} & {$[\mathrm{N}-\mathrm{m} / \mathrm{rad}]$} \\
\hline Umbilical X-axis & 25.0 & 3.0 \\
\hline Umbilical Y-axis & 25.0 & 3.0 \\
\hline Umbilical Z-axis & 50.0 & 3.0 \\
\hline
\end{tabular}




\section{H2 Control Design}

An optimal controller design using a frequency weighted linear quadratic regulator (LQR) along with a full order Kalman filter was chosen as a candidate design methodology. This facilitates the design of robust controllers for the case of multi-input multi-output (MIMO) multidegree-of-freedom (MDOF) systems. Before proceeding to the controller design for g-LIMIT a brief summary of recent research into the implementation of the $\mathrm{H} 2$ methodology to at microgravity isolation problem for a single-degree-of-freedom (SDOF) system will be presented.

\section{SDOF Case Study using Frequency Weighted H2}

A SDOF case study has demonstrated the utility of the frequency weighted LQR approach applied to the microgravity vibration isolation problem [3]. The design of this class of linear optimal controllers requires a suitable choice of the frequency weighting design filters[3]. The inherent kinematic coupling of the state variables complicates the choice of appropriate weighting functions. Indeed, certain combinations of state frequency weighting can lead to conflicting requirements for the controller optimization. This may result in poorly conditioned regulator and/or estimator Ricatti equations [3]. Recent results of a SDOF controller design study has developed a method that provides guidance in selecting state weighting filters [3]. In this research the frequency weighting filters have been related to the weighting $V_{s}$ of the pseudosensitivity function $S$ and the weighting $V_{T}$ of the pseudo-complementary-sensitivity function $T$. This technique leads to an intuitive weighting filter selection process for loop-shaping. This intuition arises from the fact that the performance index (for cheap control) can be expressed in terms of the pseudo-sensitivity and pseudo-complementary-sensitivity functions for a system having, as output, the flotor acceleration, and input, the stator acceleration (indirect disturbance). 
Thus choosing appropriate weighting stratcgies for $S$ and $T$ leads the designer to consider corresponding weighting-filter choices yielding a rational approach to filter selection. Equations (1) through (3), obtained from reference [3], show the relationship of $S$ and $T$ to the quadratic performance index $J$ and the relationship of the state weights $W_{A}$ (weighting on $x_{a}$ ), $W_{B}$ (weighting on $x_{l}$ ), and $W_{c}$ (weighting on $x_{r}$ ) to $S$ and $T$. (Note: In this section the vector notation has been omitted since this development pertains to the SDOF case) Assuming that $J$ does not contain control weighting (i.e. cheap control) one has the following equation for the integrand of the quadratic performance index $I_{J}[3]$

$$
I_{J}=a_{i n}(s)^{*}\left[S^{*} V_{S}^{*} V_{S} S+T^{*} V_{T}^{*} V_{T} T\right] a_{i n}(s)
$$

Thus $I_{J}$, and hence $J$, is determined by the sum of a weighting $V_{S}$ on the relative acceleration (i.e. stator relative to flotor) and a weighting $V_{T}$ on the absolute acceleration of the flotor. It was also shown in reference [3] that the pseudo-sensitivity function may be expressed in terms of the state weightings as:

$$
V_{S}=\left[\left(\frac{W_{A}}{s^{2}}\right)^{*}\left(\frac{W_{A}}{s^{2}}\right)+\left(\frac{W_{B}}{s^{2}}\right)^{*}\left(\frac{W_{B}}{s^{2}}\right)\right]^{\frac{1}{2}},
$$

and the pseudo complementary-sensitivity function may be expressed in terms of the state weightings as:

$$
V_{T}=W_{c},
$$

Thus, the above equations provide a basis for the choice of the state weighting filters $\left(W_{A}, W_{B}\right.$, and $W_{C}$ ) when considering the requirement trade-off between minimizing relative and absolute acceleration. The next section will discuss this trade-off in acceleration attenuation 
requirements as it applies to microgravity isolation controller design. This will lead a rational approach for selected the weighting filters.

\section{Microgravity Vibration Isolation Design Criteria}

The microgravity vibration isolation controller design problem is summarized by (1) consideration of the rattlespace requirements (i.e. bumping of flotor against stator) at low frequencies $(<0.01 \mathrm{~Hz})$ and, (2) attenuation of the absolute acceleration of the flotor at mid-range frequencies (from $0.01 \mathrm{~Hz}$ up to $10.0 \mathrm{~Hz}$ ), and (3) a "turning off" of the control effort at some high frequency say around $20-30 \mathrm{~Hz}$. The rattlespace requirement corresponds to a tracking of the flotor's motion relative to the stator for low frequencies. Since the stator motion will be significant at low frequencies (i.e. ISS motion is very large at orbital frequency), the relative acceleration between the stator and flotor should have a unit closed-loop transmissibility to indirect disturbances over the low frequency range to avoid flotor to stator contact. To meet the science requirements the absolute flotor acceleration transmissibility should be attenuated by three orders-of-magnitude with frequency roll-off of $20 \mathrm{db} / \mathrm{dec} a d e$ over a range from $0.01 \mathrm{~Hz}$. to $10 \mathrm{~Hz}$. Above this frequency the controller should "turn-off" and the closed-loop transmissibility should rejoin the open-loop transmissibility. This will avoid excitation of any high frequency vibrational modes. The above design criteria should be meet while limiting the actuator control effort to less than $40 \mathrm{amps} /$ mirco-g over the entire frequency range [3].

Using the above design criteria along with Equations (1) through (3) a rational approach to selecting the state weighting filters can be developed: [3]. A summary of this criteria from reference [3] follows:

State Weighting Design Criteria 
(1) Use the relative-position and relative-velocity state weighting to shape the low frequency closed-loop acceleration transmissibility to control relative acceleration.

(2) Use the acceleration state weighting to shape the closed-loop acceleration transmissibility to attenuate mid-range frequencies.

(3) All state weighting filters should be chosen to cause adequate roll-off of $S$ and $T$ at high frequencies, thus forcing the control to "turn-off".

The state frequency weighting LQR approach described above was applied to the SDOF system in a case study to determine the performance of the $\mathrm{H} 2$ methodology [3]. Four different scenarios involving selection of the state weighting filters, consistent with the approach described herein, were investigated in the study. Numerous observations of the effects of state wieghting, measurement noise, and process noise parameters on the frequency shaping of the acceleration transmissibility as they relate to the regulator and observer designs are described in this reference. Explainations are provided that relate the observations to the effect on $S$ and $T$. These case studies provides good examples of the logical application of the methodology and demonstrate good performance in meeting the the design. Turn now to the application of this state weighting filter selection process to the $6 \mathrm{DOF}$ vibration control for g-LIMIT using the $\mathrm{H} 2$ methodology.

\section{g-LIMIT 6DOF Case Study}

A rational approach to state frequency weighting filter selection for the $\mathrm{H} 2$ control design method, summarized in the previous section, has been developed, justified, and demonstrated for the SDOF system in the design case studies [3]. This method will be applied to the vibration 
isolation of 6DOF system for g-LIMIT in an attempt to ascertain the feasibility of extending this technique to MDOF controller design. Acceleration responses to rotational and translational, direct and indirect disturbances will be investigated. Closed-loop system acceleration transmissibility will be compared to the open-loop responses to demonstrate fulfillment of the design criteria for the nominal plant characteristics. The system robustness to modeling errors will be analyzed by investigating the effects of changes in the umbilical stiffness on closed-loop performance.

\section{Measurement Selection}

Feed back of the absolute acceleration of the flotor will be used for the 6DOF controller designs. As stated in reference [3], any controller which uses only acceleration feedback to attenuate indirect disturbances will cause attenuation of direct disturbances. This is a result of the increase effective mass from an acceleration-only feedback controller. Thus, the choice of acceleration-only feedback allows the designer to focus on attenuation of indirect disturbances while attenuation of direct disturbances will be realized as a consequence. This increase in effective mass has an additional advantage of improving the stability robustness since the damping ratio increases with mass. Addition of a separate low frequency $(<0.01 \mathrm{~Hz})$ relativeposition controller can be used to meet the rattle-space requirement. The controller design presented herein will focus on attenuation of acceleration transmitance and will not include a separate controller to meet the rattle-space objective.

Weighting Filter Selection 
The rational for selecting the state weighting filters for the $\mathrm{H} 2$ optimization is to select the desired loop shaping of the pseudo-sensitivity function $S$ and the pseudo-complementarysensitivity function $T$. This desired shaping is then related to the state weighting filter selection and to the acceleration control objectives as specified by design criteria $\# 1, \# 2$, and \#3 in the above section. To meet design criteria \#1 the specification of relative-position and relativevelocity state weighting is selected to provide a unit acceleration transmissibility at low frequencies $(<0.01 \mathrm{~Hz}$.). This corresponds to relatively high weighting on $\mathrm{S}$ at low frequencies which diminishes considerably at mid-range and high frequencies. To meet design criteria \#2 the specification of acceleration state weighting is selected to provide the required attenuation of the acceleration transmissibility at mid-range frequencies (between $0.01 \mathrm{~Hz}$. and $10 \mathrm{~Hz}$.). This corresponds to low weighting on $T$ at low frequencies which increases over the mid-range and diminishes considerably over high frequencies. Design criteria \#3 is accomplished by selecting all state weightings to attenuate $S$ and $T$ over the high frequency range. With these objectives in mind a good choice of state weightings would result in a low-pass shaping of $S$ with corner frequency about $0.01 \mathrm{~Hz}$, and a band-pass frequency shaping of $S$ with pass band over the midrange frequencies. Alternatively, an integrating-type frequency shaping of $S$ could be used in lieu of the low-pass shaping since the desired comer frequency is very low. The advantage would be that weighting of $S$ over quasi-static frequencies would be increased substantially. This would aide the controller in meeting criteria \# I while additionally creating frequency separation of the inherently conflicting criteria \#1 and \#2.

In the SDOF case studies [3] the above strategy for selecting the state weighting was used in controller design scenarios \#3 and \#4. Relatively high weighting on $T$ was used in these cases in which case it was found that the regulator dominated the closed-loop response (i.e. the 
resulting response did not vary significantly as a result of including a Kalman filter for state reconstruction). This regulator dominance seems to result in a more intuitive tuning process of the closed-loop response since the design criteria are related to the LQR performance index. Using this approach a controller design with weighting filter selection similar to the SDOF scenarios \#3 and \#4 was chosen for the MDOF case study.

\section{g-LIMIT MDOF Design}

Figure 1 shows a plot of the state weighting design for the g-LIMIT MDOF case study. Each translational and rotation degree-of-freedom uses the same respective state weighting. Band-pass filters (with consecutive legs having slopes $+1,0,-2$ ) on the each absolute acceleration state, flat filters (i.e. constant weightings) on each relative-position state, and open filters on the relative-velocity states were used for the translational degrees-of-freedom. Flat filters, with the same wieghting magnitude as the relative-position states, were used for the relative-angular-position states, along with open filters for the relative-angular-velocity states. Control weightings were constant with a magnitude of 100 for each actuator. Figure 2 shows the corresponding weighting on the the pseudo-sensitivity function and the pseudo-complementarysensitivity function.

Analysis of the closed-loop performance of the MDOF controller begins with consideration of the attenuation of indirect acceleration disturbances. Figures 3, 5, and 7 show the Open Loop (OL) and Closed Loop (CL) transmissivities to indirect disturbances $\underline{a}_{i n}$ for each component direction. The SDOF Case 3 from reference [3] was used to as a template for the design. In this case the attenuation of indirect disturbances met the specification but the CL transmissibility rejoined the OL plot above a frequency of $10 \mathrm{E} 5 \mathrm{~Hz}$. This is considered 
undesirable since the controller inputs energy into the system at high frequencies and does not "turn-off" as required by design criteria \#3. Lowering the pass band of the acceleration weighting filter to between 0.001 and $0.75 \mathrm{~Hz}$ and decreasing the slope of the third leg of this filter from -1 to -2 alleviated this problem. The desired "turn-off" frequency was achieved by adjustment of the filter magnitude in the pass band. The CL transmissibility rejoined the OL at a frequency of approximating $20 \mathrm{~Hz}$. The desired break frequency of $0.01 \mathrm{~Hz}$ was achieved by adjusting the relative weighting on $S$ and $T$, and using a relatively low value of measurement noise (acceleration noise variance $=1 \mathrm{E}-6 \mathrm{~m} / \mathrm{sec}^{\wedge} 2$ ). Relatively high weighting on $S$ for low frequencies resulted in the desired unit transmissibility for quasi-static disturbances. As shown in Figures 3, 5, and 7 the design criteria for indirect disturbance attenuation was met for on-axis repsonses (i.e. in the same direction as the disturbance loading). Furthermore, the off-axis OL transmissibility was attenuated over the mid-range frequencies producing a necessary reduction in the resonant amplitude of the off-axis flotor vibration modes. The off-axis transmissibility was somewhat increased over the low frequency range but considered acceptable since it was at least two orders-of-magnitude less than the on-axis response over this range. The actuator current values necessary for indirect disturbance attenuation were maintained at levels less than 1 ampere/micro-g over the entire frequency range for all disturbance directions as shown in Figures 4,6 , and 8 . In some cases, particularly noticeable in the $\mathrm{Z}$ direction disturbance loading repsonses shown in Figure 7 , the off-axis CL transmissibility did not rejoin the OL plot at the desired frequency. This caused an increase in the control effort at high frequency as shown in Figure 8. However, since the control effort was maintained well below $1 \mathrm{E}-10 \mathrm{Amp} / \mathrm{micro}-\mathrm{g}$ over this frequency range the increase is considered negligible at it pertains to actual system performance. 
Turning now to the attenuation of direct acceleration disturbances. Figures 9, 11, and 13 show the OL and CL transmissibilities to direct disturbances $\underline{a}_{t}$ for each component direction. The corresponding actuator current values are shown in Figures 10, 12, and 14. As predicted, accomplishing the goal of the indirect disturbance attenuation has the desirable consequence of direct disturbance attenuation over the mid-range frequencies. As shown by the plots of direct disturbance transmissibility this holds somewhat for off-axis as well as on-axis responses. The CL off-axis responses are increased over the low frequency range as was the case with the indirect disturbance loadings. Again, the off-axis CL transmissibility is considered acceptable since it is at least two orders-of-magnitude below the on-axis CL response for each loading direction. The on-axis attenuation is about three orders-of-magnitude at the flotor resonance frequency for each loading direction. Additionally, the off-axis responses are attenuated significantly over the mid-range frequencies. For direct disturbance loading there are relatively low demands on the actuator currents over the entire frequency range.

Turning to the attenuation of direct rotational acceleration disturbances. Figures $15,17$. and 19 show the OL and CL transmissibilities to direct rotational disturbances $\underline{\alpha}_{d}$ for each component direction. The corresponding actuator current values are shown in Figures 16, 18, and 20.

\section{Design Robustness}

The $\mathrm{H} 2$ Controller design robustness was evaluated by consideration of umbilical stiffness modeling error. Several cases were analyzed in which the diagonal stiffness values for each umbilical were allowed to vary over a wide range. Additionally, the individual umbilical coordinate systems (the coordinate directions for diagonalizing the stiffness matrices) were 
misaligned by up to $40 \mathrm{deg}$ from the stator-fixed coordinate directions. This resulted in significant off-diagonal terms in the system translation and rotational stiffness matrices. The results of one of these cases are presented in this section to demonstrate the $\mathrm{H} 2$ controller robustness to umbilical stiffness modeling errors. The umbilical modeling error used in this robustness test case are summarized by the following.

(1) The diagonal umbilical stiffness values were increased by $50 \%$ over those used for the design.

(2) The umbilical coordinate systems were misaligned by $-20,+20$, and +40 deg. respectively, from the orientations used for the design.

Figures 21, 23, and 25 show the indirect-, direct-, and direct-angular- disturbance transmissibilities, respectively, for a $Z$ direction/axis loading using the modified system equations with the original, unmodified $\mathrm{H} 2$ controller. The other loading directions, not shown herein, produced similar results for this modeling error. A significant increase in the off-axis OL transmissibilities occurred, for each type of loading, as a result of the misalignment of umbilical stiffness directions with the stator-fixed directions. An increase in the flotor's resonant frequency resulted from the $50 \%$ increase in the diagonal stiffness values. The unmodified controller performed very well in lieu of this relatively severe modeling error. The transmissibility to indirect disturbance, shown in Figure 21, was mostly unaffected by the modeling error. Notable exceptions are that the controller roll-off frequency increased slightly above $0.01 \mathrm{~Hz}$ and the low frequency $\mathrm{CL}$ off-axis responses increased by an order-of-magnitude. The increase in the low frequency CL off-axis response appears to be characteristic of this design approach. Apparently, this increase corresponds with the unavoidable increase in the OL off-axis resonant mode amplitude. Even with this modeling error the $C L$ off-axis responses are acceptable since they are 
significantly lower than the on-axis transmissibilities over the entire frequency range. Good attenuation of the transmissibility to direct disturbance loading is apparent in Figures 23 and 25. The controller is apparently very robust to the increase in off-axis transmissibility at the flotor resonant frequency as was the case with the indirect-disturbance loading. Figures 22, 24, and 26 show the actuator current demands for the associated disturbance loading conditions. The increase in off-axis umbilical stiffness resulted in an increase in the $\mathrm{X}$-Y actuator current demand for each type of disturbance loading. However, the maximum current demand over all frequencies remained less than $1 \mathrm{Amp} / \mathrm{milli}-\mathrm{g}$.

The robustness of the $\mathrm{H} 2$ controller was evaluated over a wide range of diagonal stiffness values and umbilical-to stator-frame misalignment angles. Adequate performance was achieved over a range of diagonal stiffness modeling error from about $-50 \%$ to $+200 \%$ and a range of $+/$ 45 deg. misalignment angles. System stability was maintained for all the simulated test cases which had diagonal stiffness modeling errors ranging from $-70 \%$ to $+500 \%$ and misalignment angles ranging between $+/-60 \mathrm{deg}$. Overall the $\mathrm{H} 2$ control design using frequency weighting performed well. System performance and stability robustness to changes in umbilical stiffness was demonstrated for the test cases executed using a wide range of umbilical stiffness values.

\section{Concluding Remarks}

This paper has presented the design of a microgravity vibration isolation controller for the Glovebox Integrated Microgravity Isolation Technology (g-LIMIT). A steady-state $H_{2}$ optimal control methodology were developed and the performance evaluated for a set of nominal

g-LIMIT system parameters using a full-order 6DOF linear state model [1]. A rational approach to selecting the state weighting filters, previously developed and demonstrated in a SDOF case 
study [3], was shown to achieve similar results for the MDOF, multi-axis system. This intuitive approach steers the designer's choice of frequency weighting. These appropriate state frequency wieghtings avoid the pitfalls of arbitrary choices that may result in conflicting requirements of the Riccati equation solution and subsequent poor solution conditioning.

The $H_{2}$ MDOF designs developed using this strategy were relatively straightforward to "tune." The final controllers performed well in all disturbance loading conditions, easily accomplishing the design objectives. Closed loop off-axis transmissibility to indirect disturbances was increased somewhat over low frequency ranges but generally held to two orders-of-magnitude below the on-axis response, and thus considered acceptable. On-axis CL responses to each disturbance loading were shown to meet the design criteria and to demonstrate robust performance to umbilical stiffness modeling errors. Moderate control effort was needed to achieve the desired CL performance with peak actuator current demands less that 1 ampere/milli-g in all cases.

Overall the $\mathrm{H}_{2}$ control design method using the frequency weighting selection criteria discussed in this paper shows promise in meeting stringent microgravity isolation design requirements. Future work should include simulation studies using non-linear system equations with high fidelity models of sensor and actuator characteristics. This will then provide an accurate picture of the actual expected system performance and may lead to controller improvements.

\section{Acknowledgments}

The authors are grateful to Marshall Space Flight Center and the National Aeronautics and Space Administration for their partial funding of this work. 


\section{References}

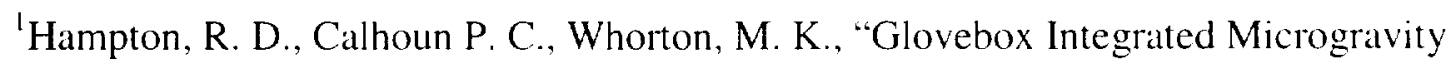
Isolation Technology (g-LIMIT): A Linearized State-Space Model," submitted as a NASA TM, June 2001.

${ }^{2}$ Whorton, M. S., et. all, "Design Definition Document (DDD) For Glovebox Integrated Microgravity Isolation Technology (g-LIMIT) Characterization Test," g-LIMIT-DOC-000I, NASA MSFC, September 30, 1999.

${ }^{3}$ Hampton, R. David, and Whorton, Mark S., "Design-Filter Selection for H2 Control of Microgravity Isolation Systems: A Single-Degree-of-Freedom Case Study," AIAA-2000-3955, August 2000. Submitted to the Journal of Guidance, Control, and Dynamics, September 2000. 


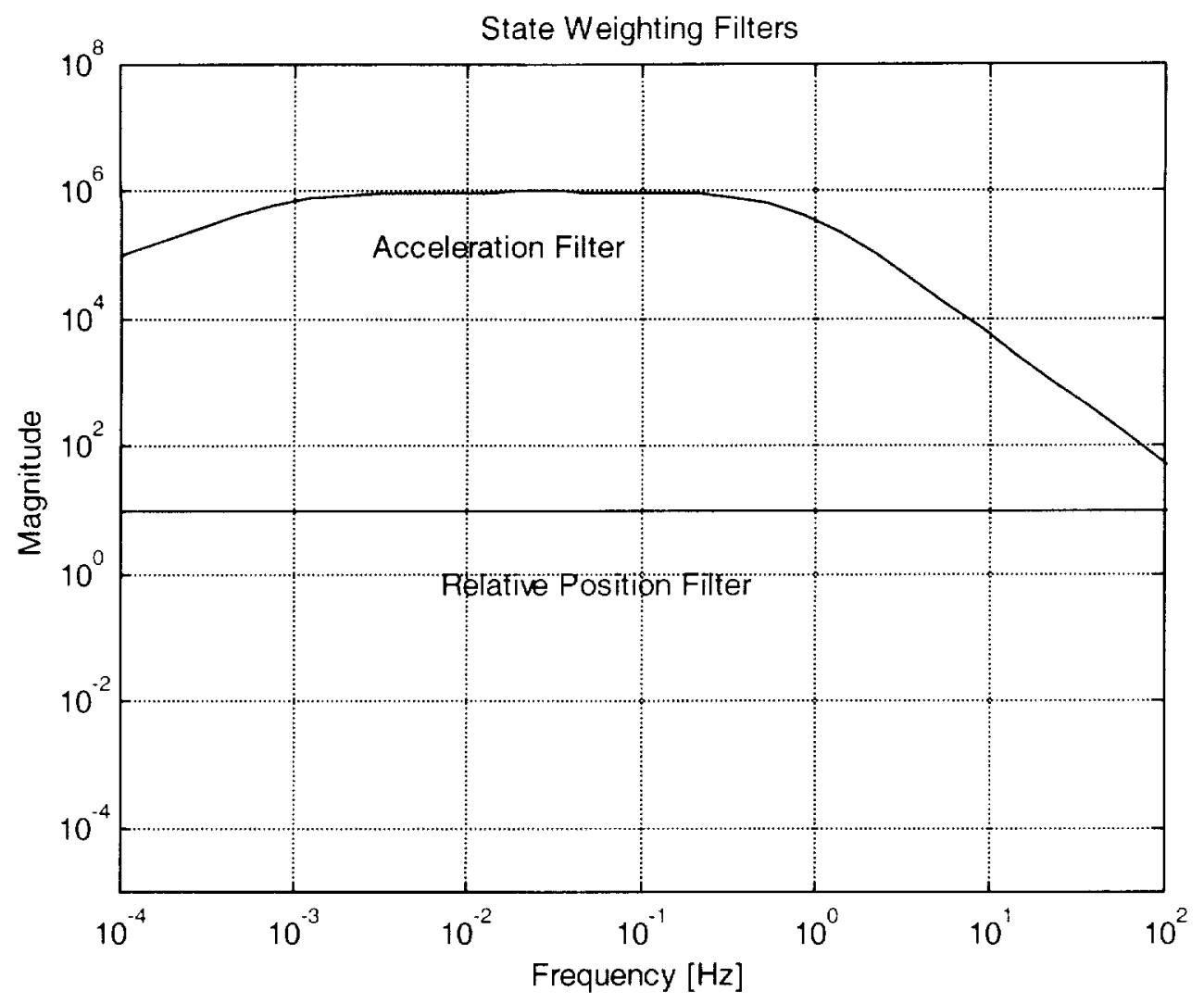

Figure 1 - Design Weighting Filters (All Degrees of Freedom) (H2 Controller)

Filters $W_{S}$ for $S$ (Sens. fnc.) and $W_{T}$ for $T$ (Comp. sens. fnc.)

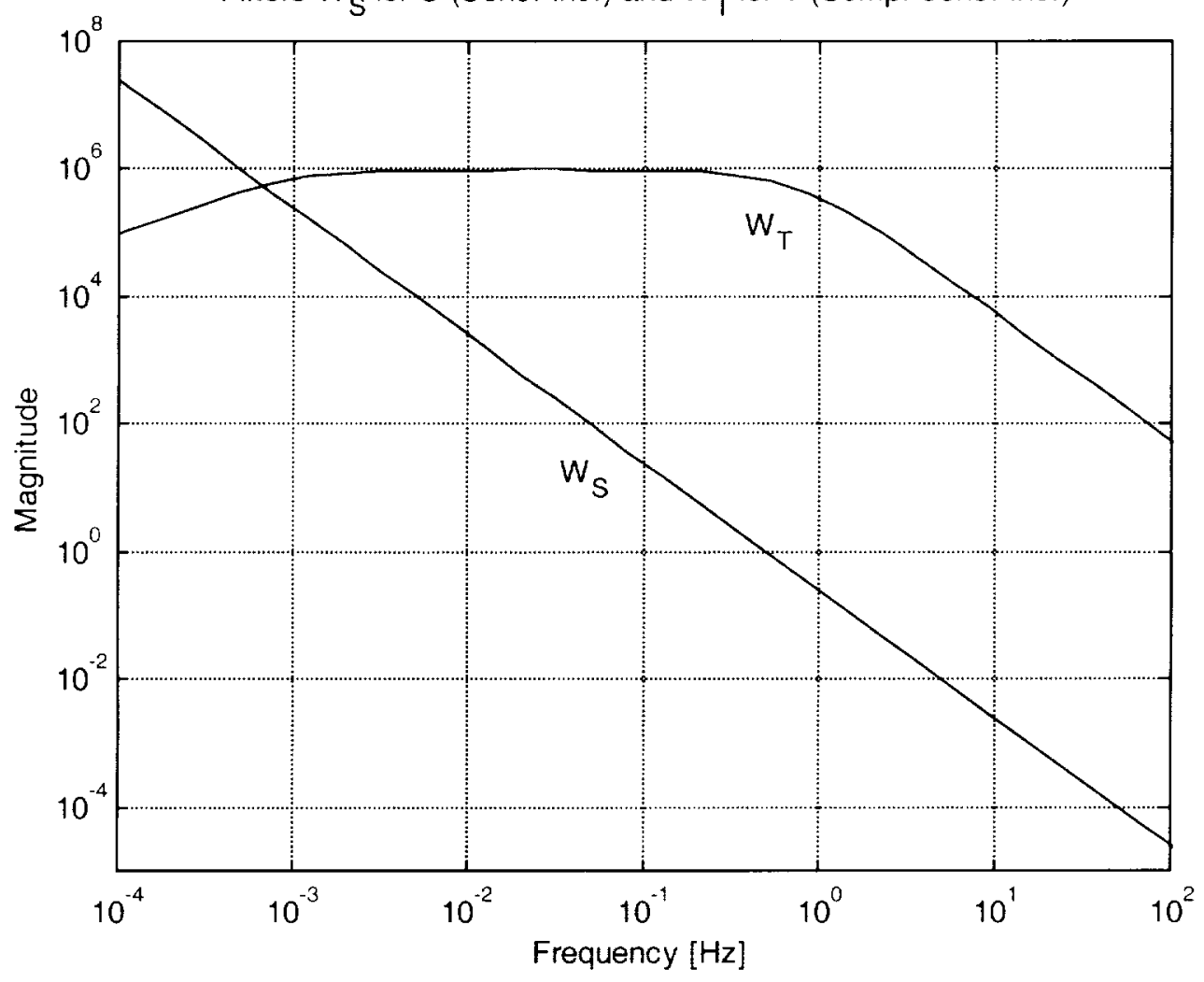

Figure 2 - Pseudo-Sensitivity- and Psuedo-Complementary-Sensitivity Function Weighting (H2 Controller) 


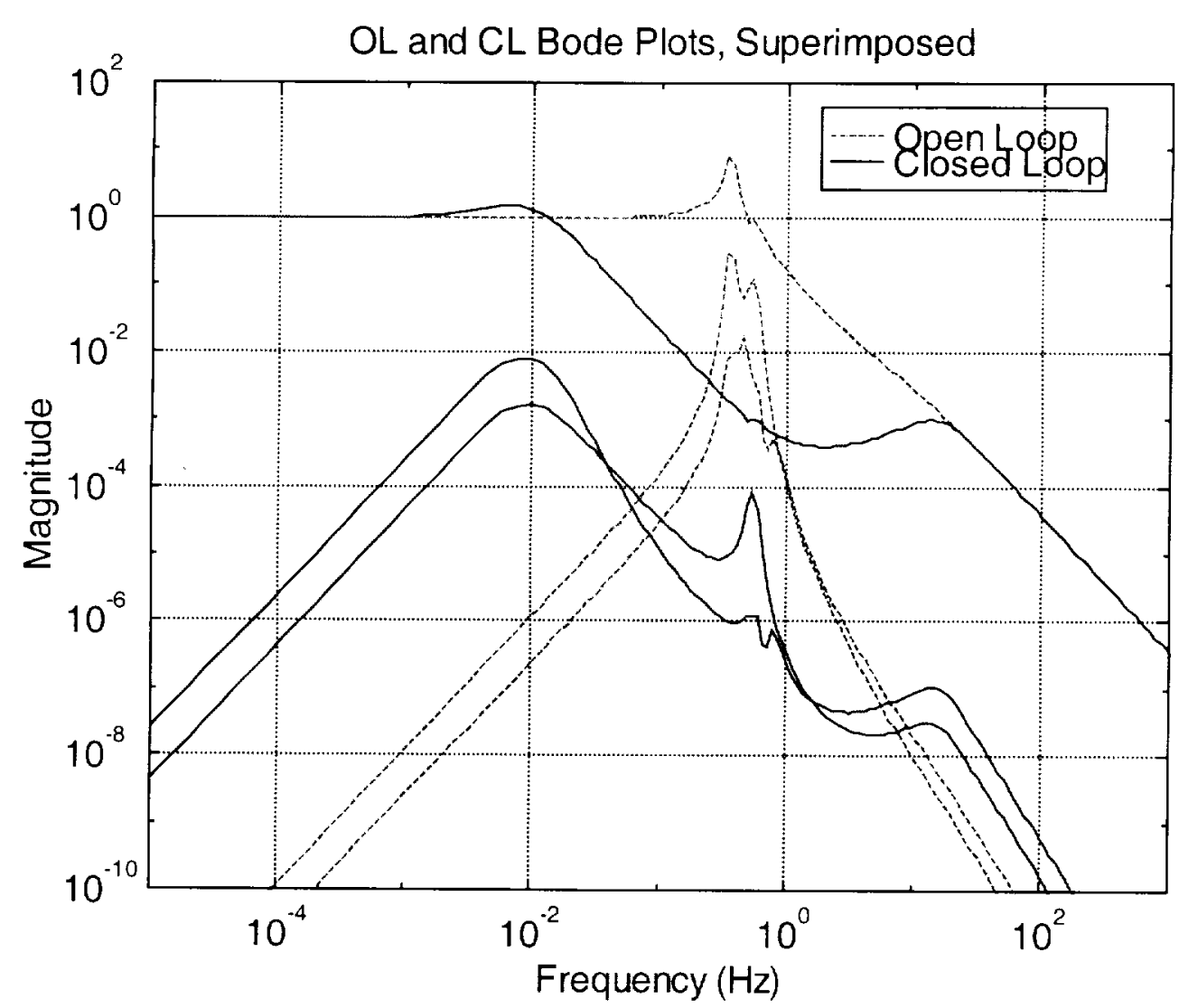

Figure 3 - Open and Closed Loop Transmissibilities for $\mathrm{X}$-Axis Indirect Acceleration Disturbances (H2 Controller)

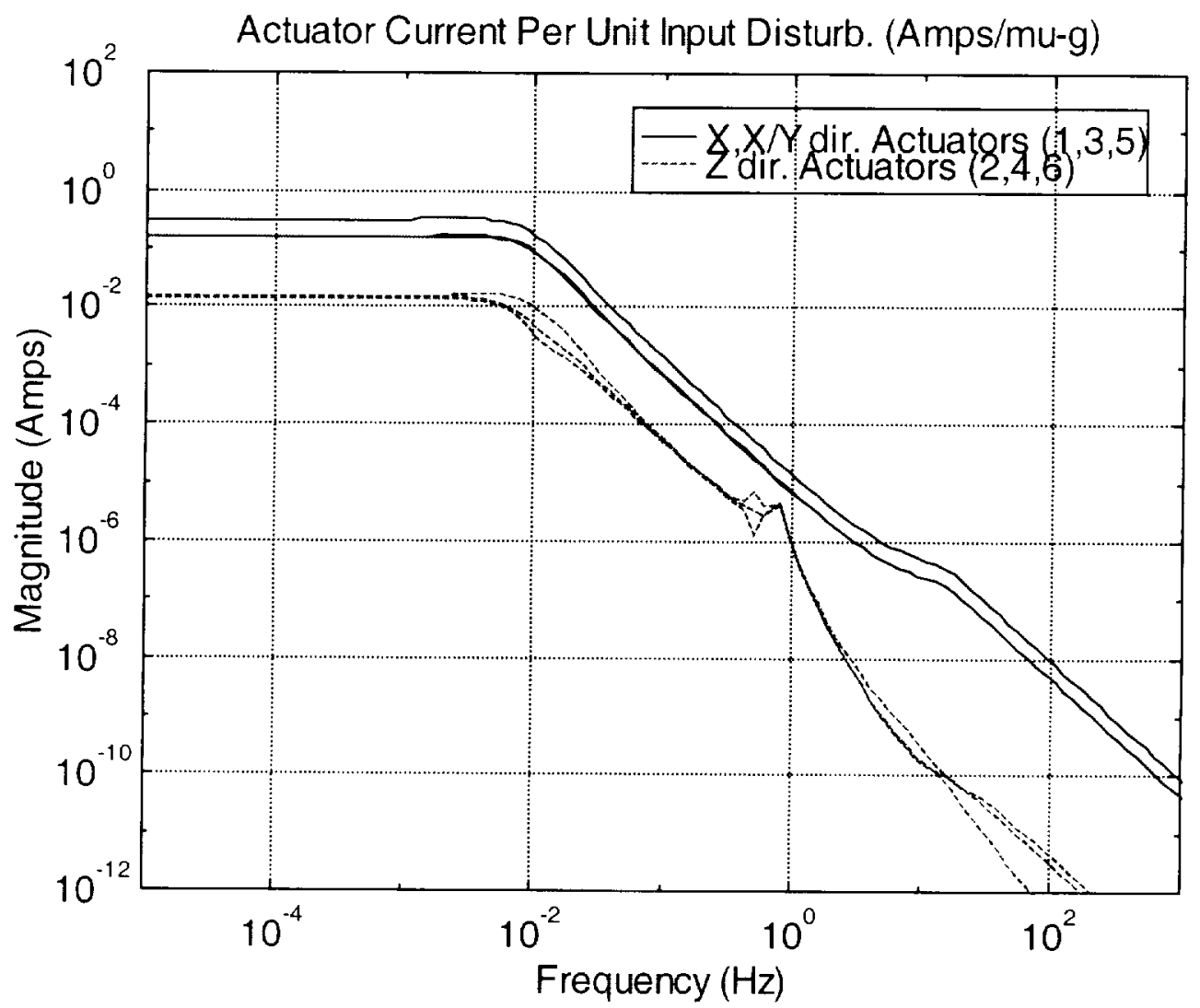

Figure 4 - Closed Loop Current Vs. Frequency

$\mathrm{X}$-Axis Indirect Acceleration Disturbances (H2 Controller) 


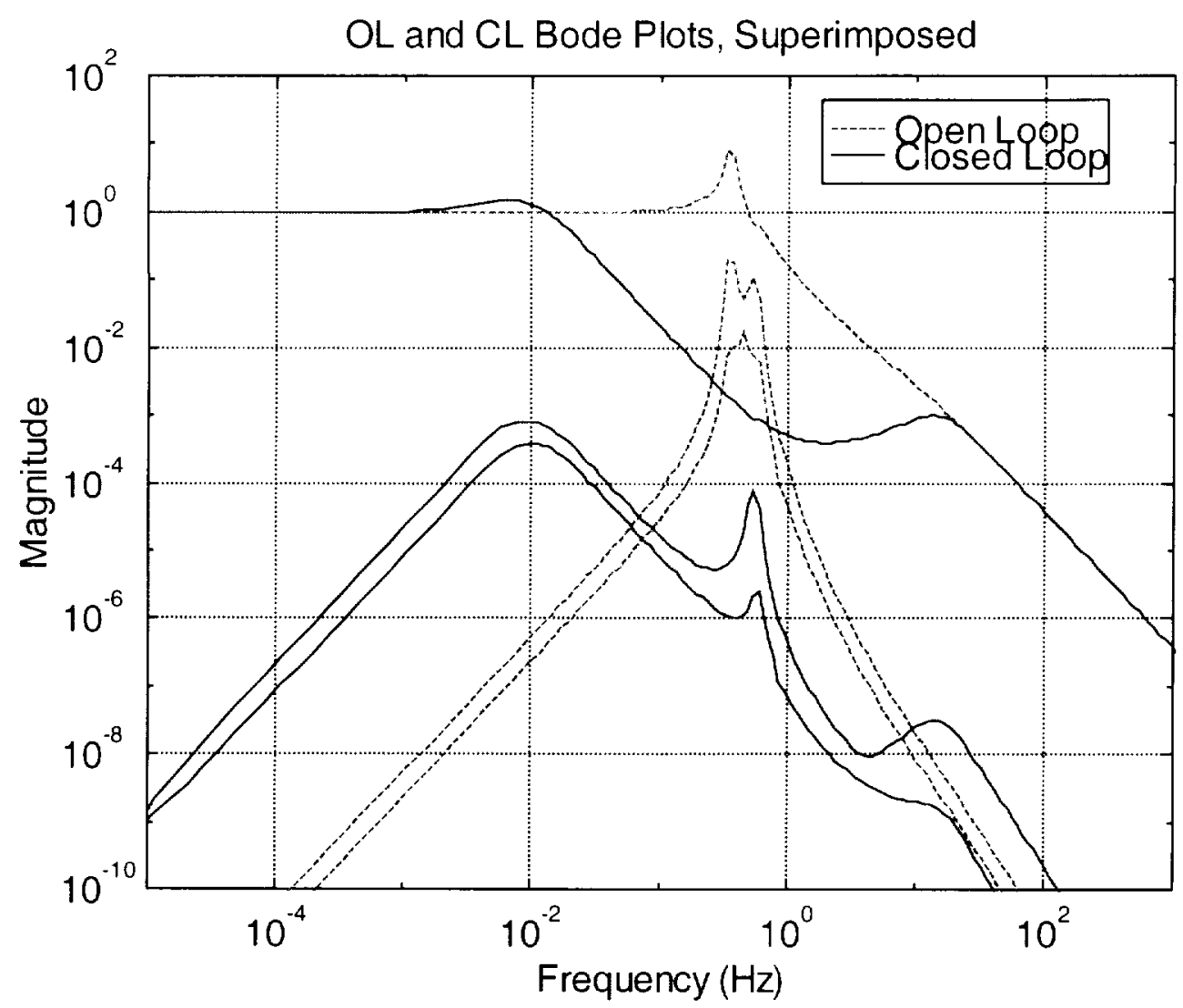

Figure 5 - Open and Closed Loop Transmissibilities for Y-Axis Indirect Acceleration Disturbances (H2 Controller)

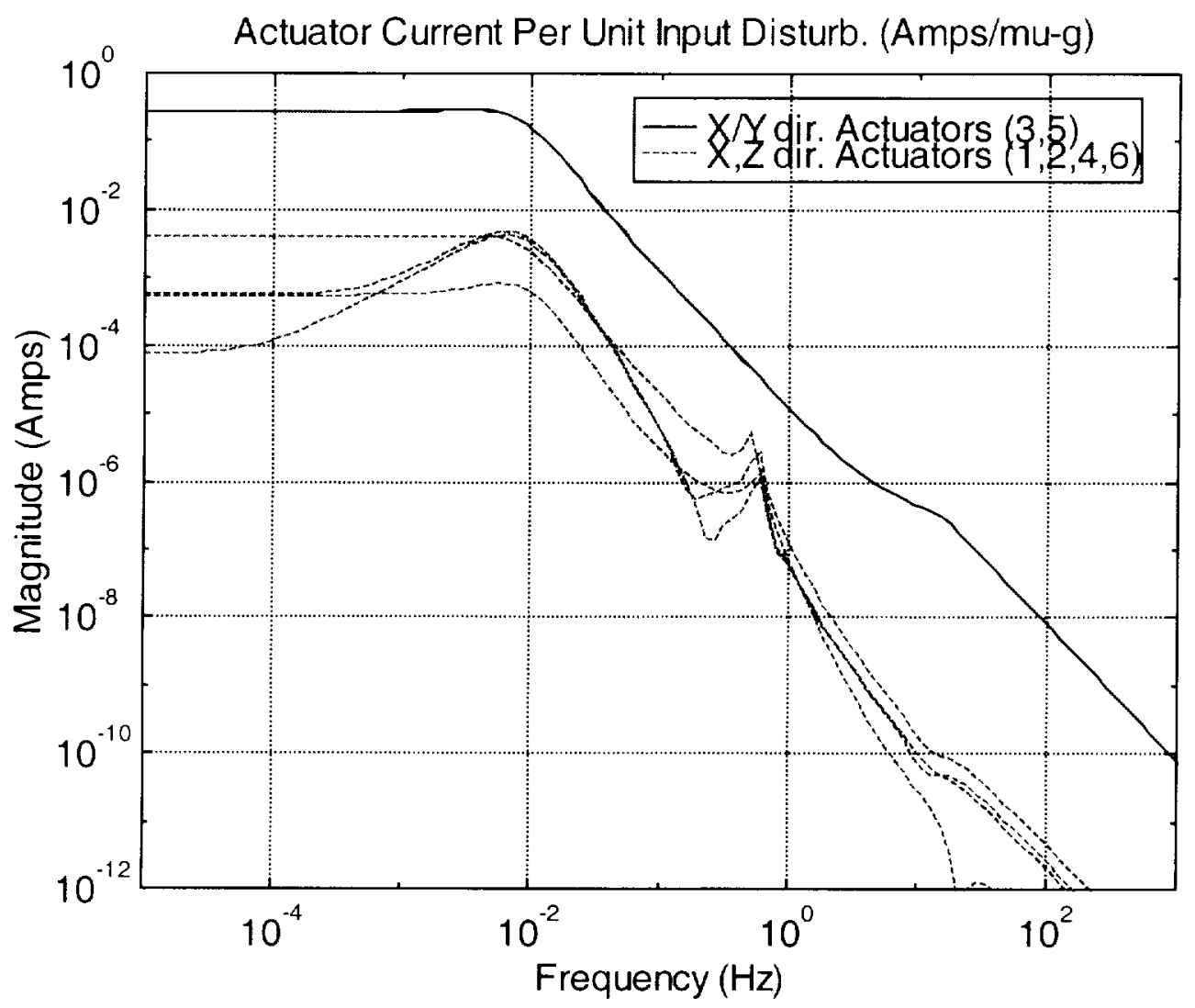

Figure 6 - Closed Loop Current Vs. Frequency Y-Axis Indirect Acceleration Disturbances (H2 Controller) 


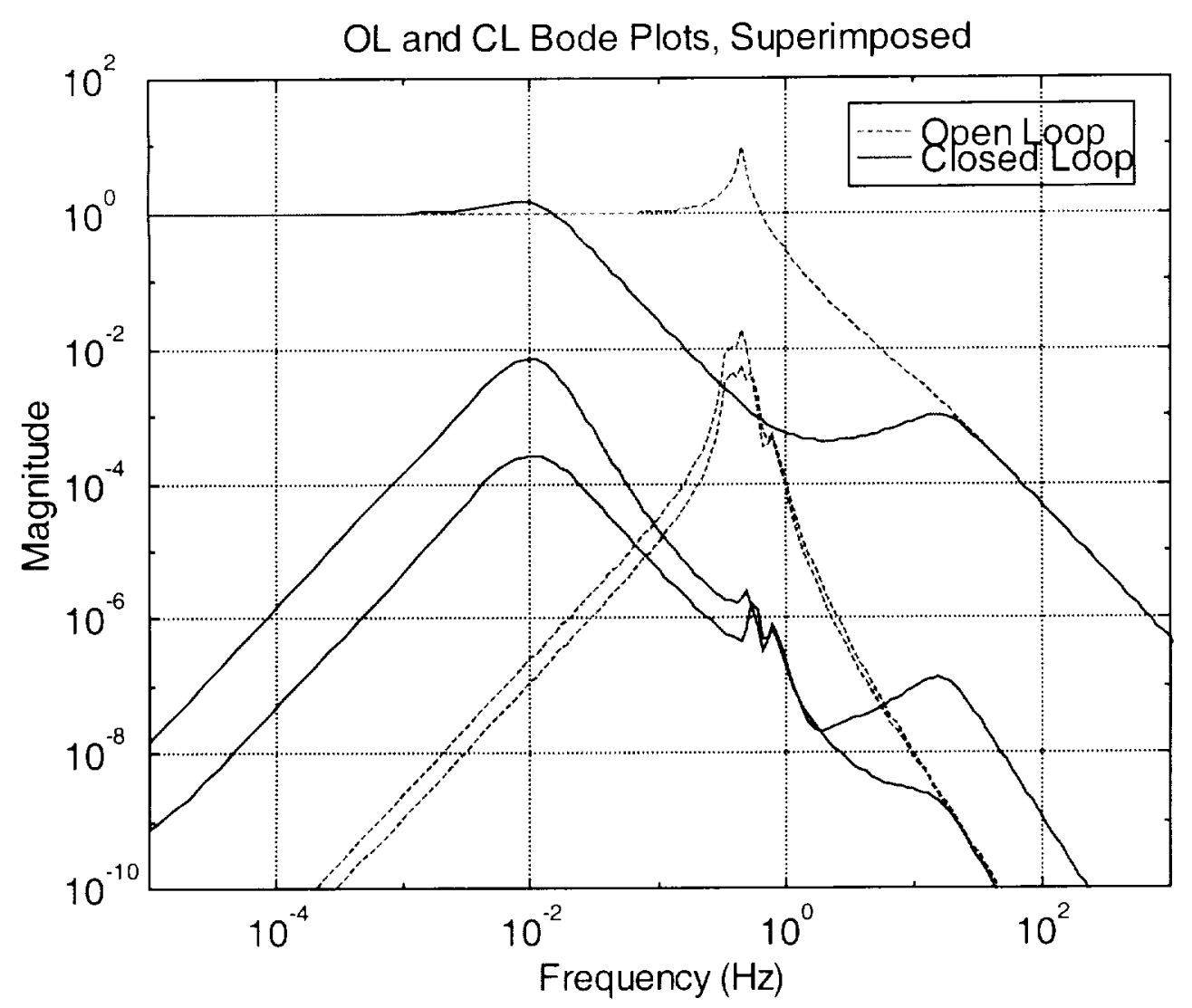

Figure 7 - Open and Closed Loop Transmissibilities for $\mathrm{Z}$-Axis Indirect Acceleration Disturbances (H2 Controller)

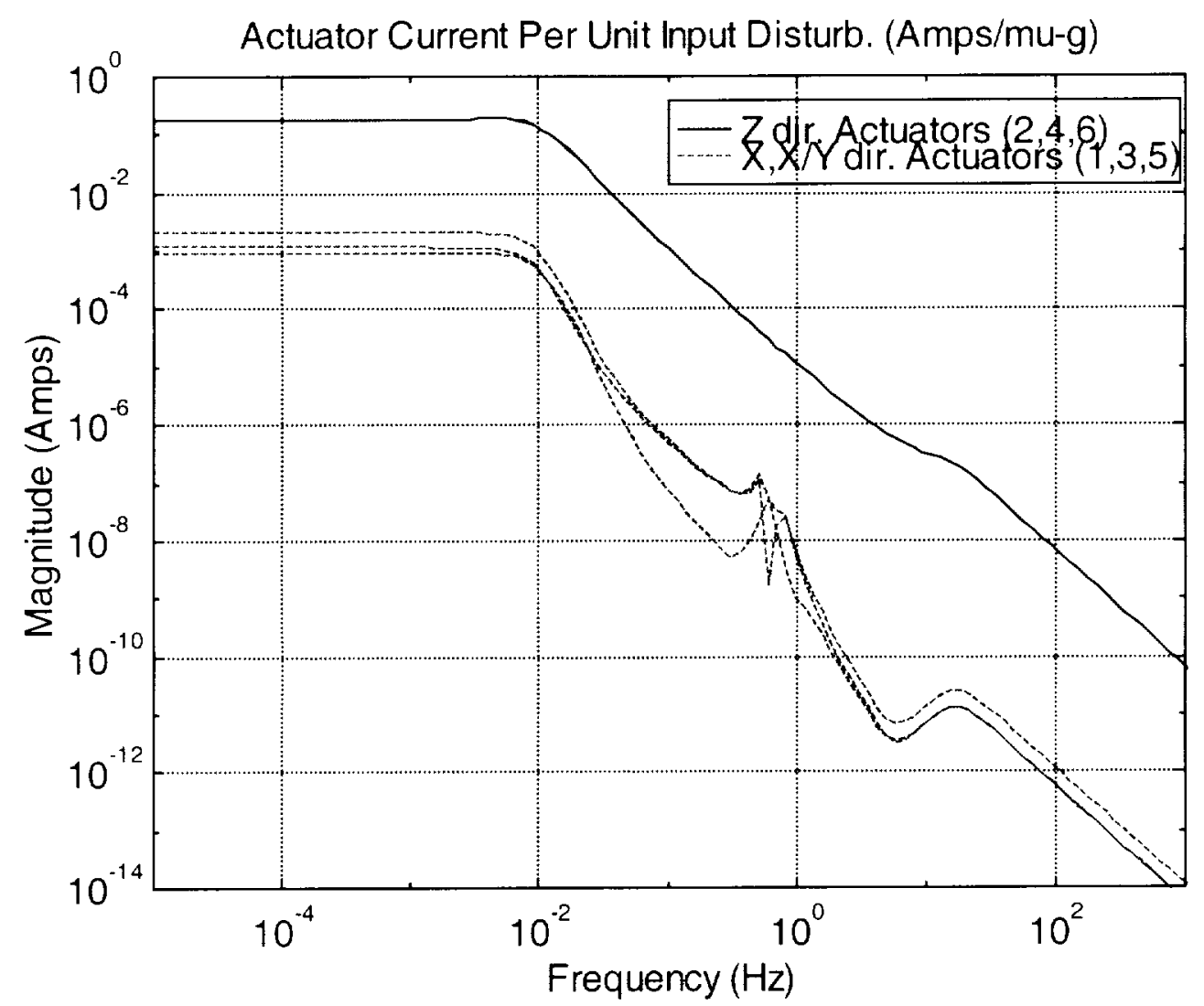

Figure 8 - Closed Loop Current Vs. Frequency Z-Axis Indirect Acceleration Disturbances (H2 Controller) 


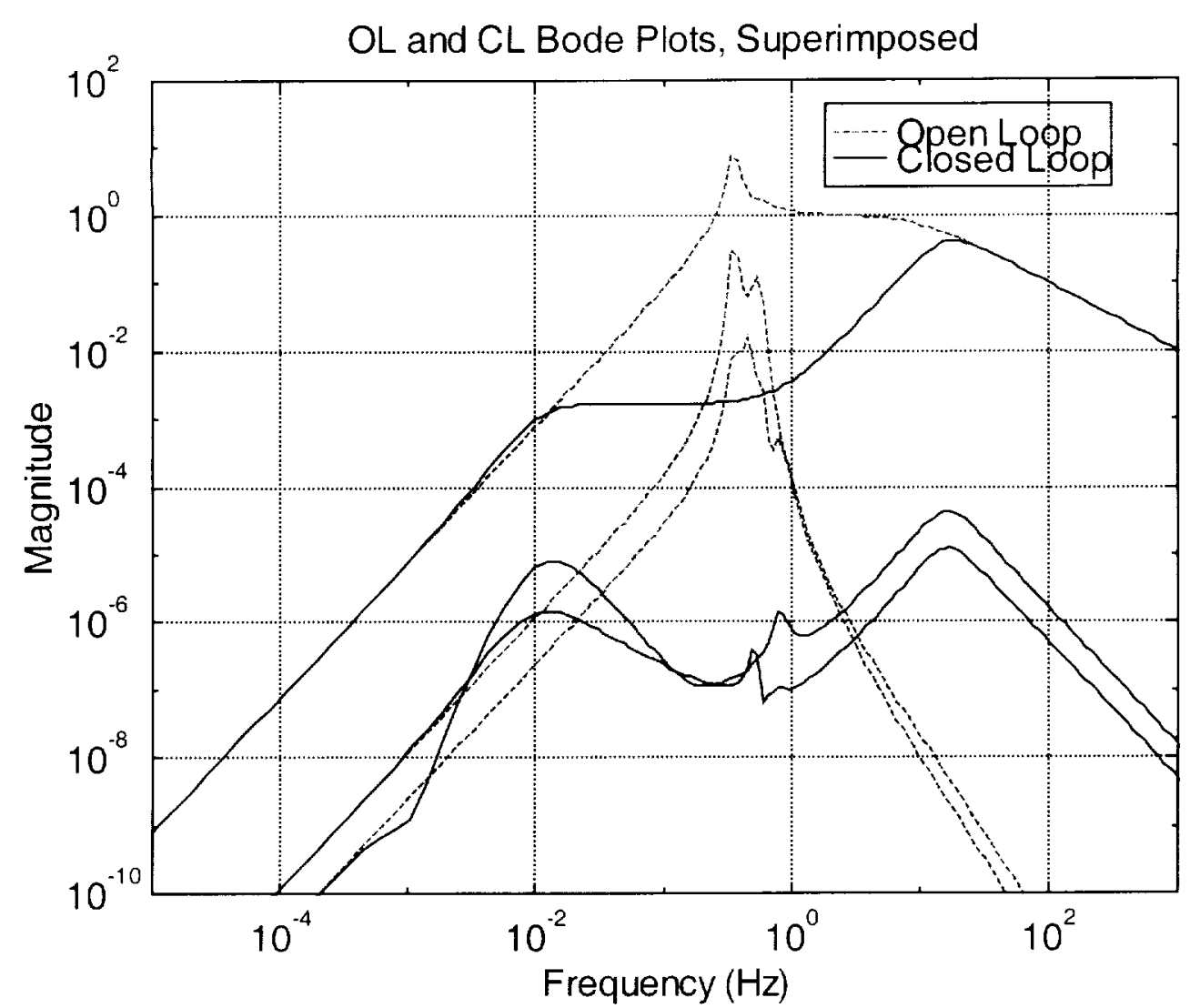

Figure 9 - Open and Closed Loop Transmissibilities for $\mathrm{X}$-Axis Direct Acceleration Disturbances (H2 Controller)

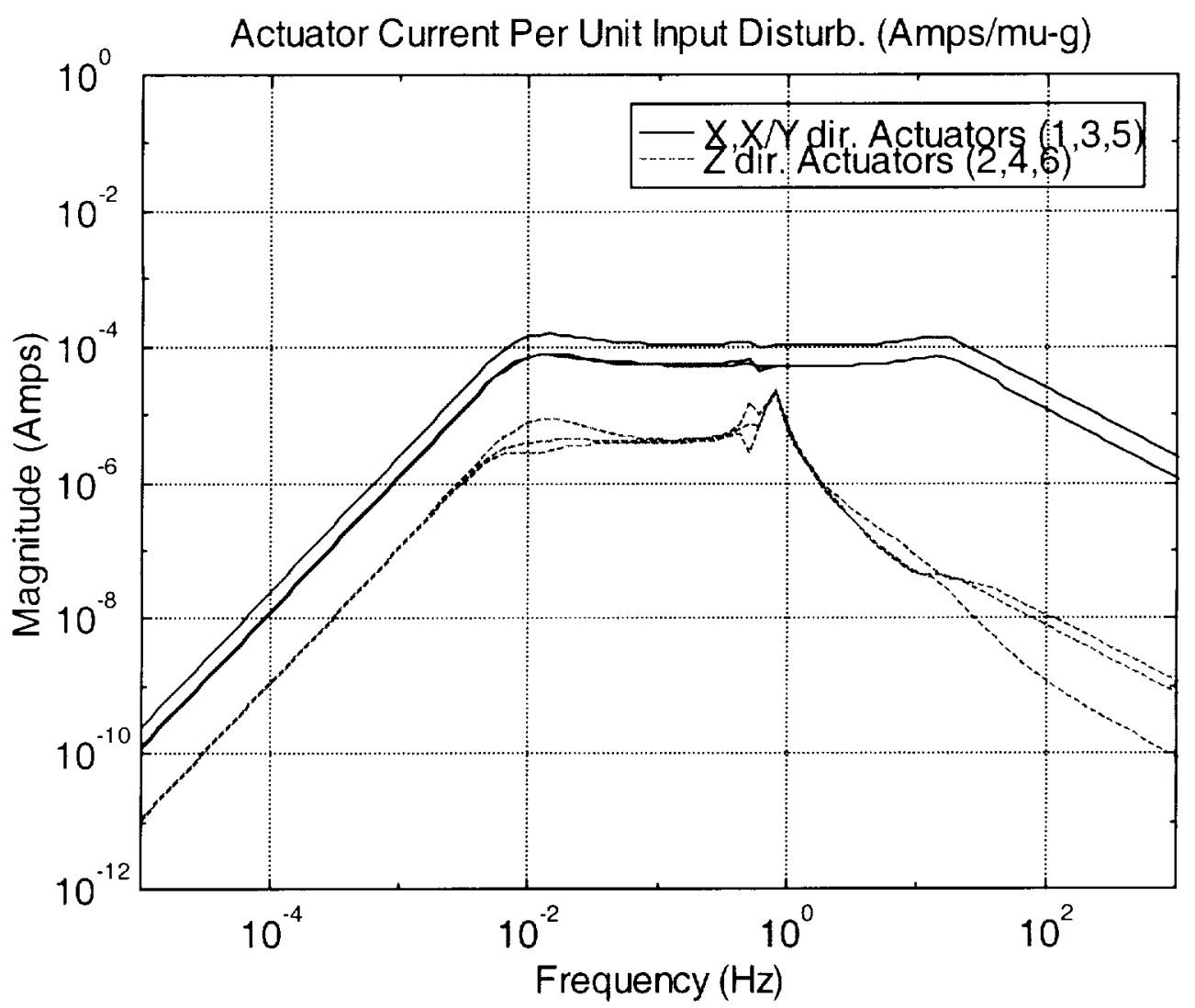

Figure 10 - Closed Loop Current Vs. Frequency $\mathrm{X}$-Axis Direct Acceleration Disturbances (H2 Controller) 


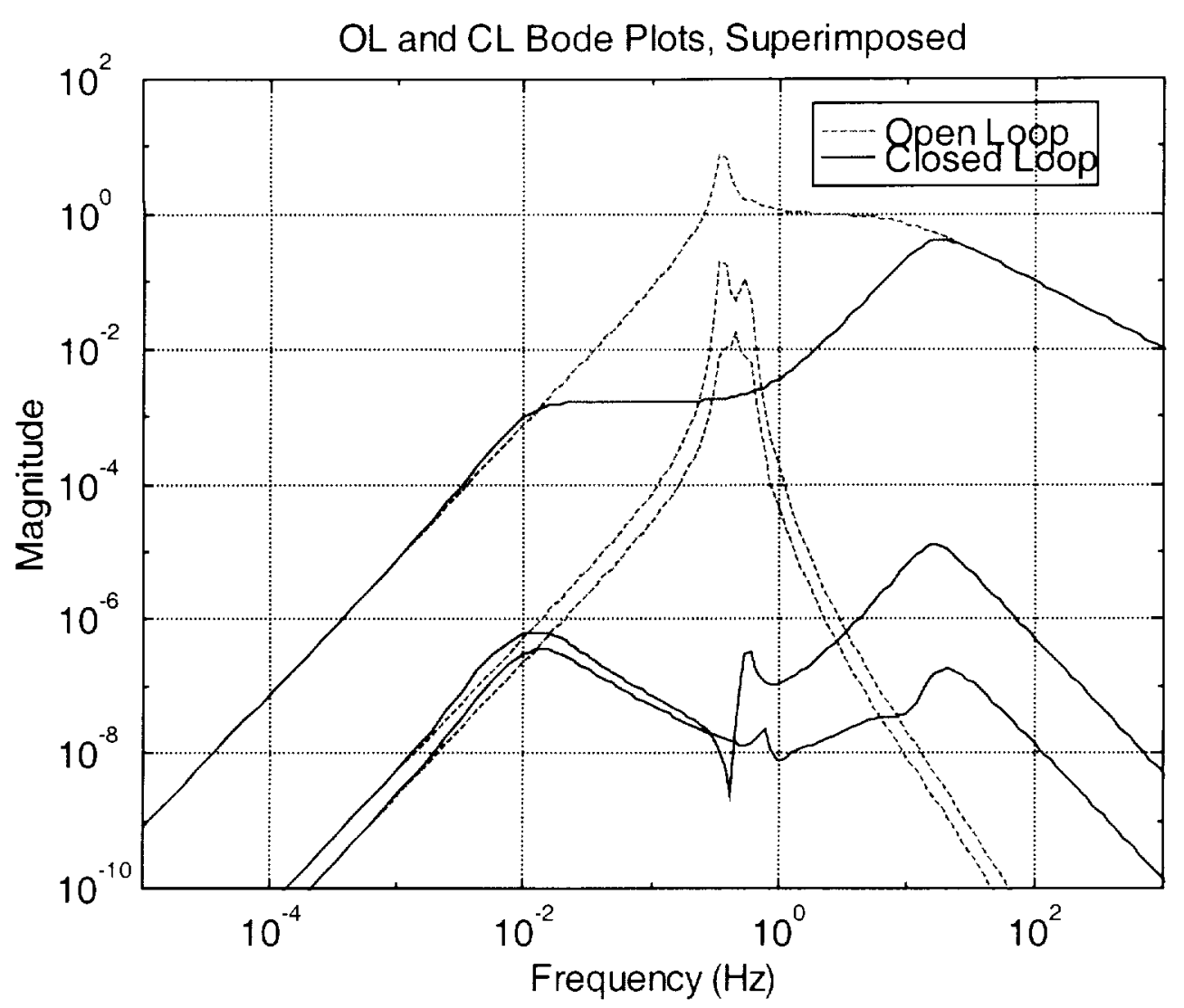

Figure 11 - Open and Closed Loop Transmissibilities for Y-Axis Direct Acceleration Disturbances (H2 Controller)

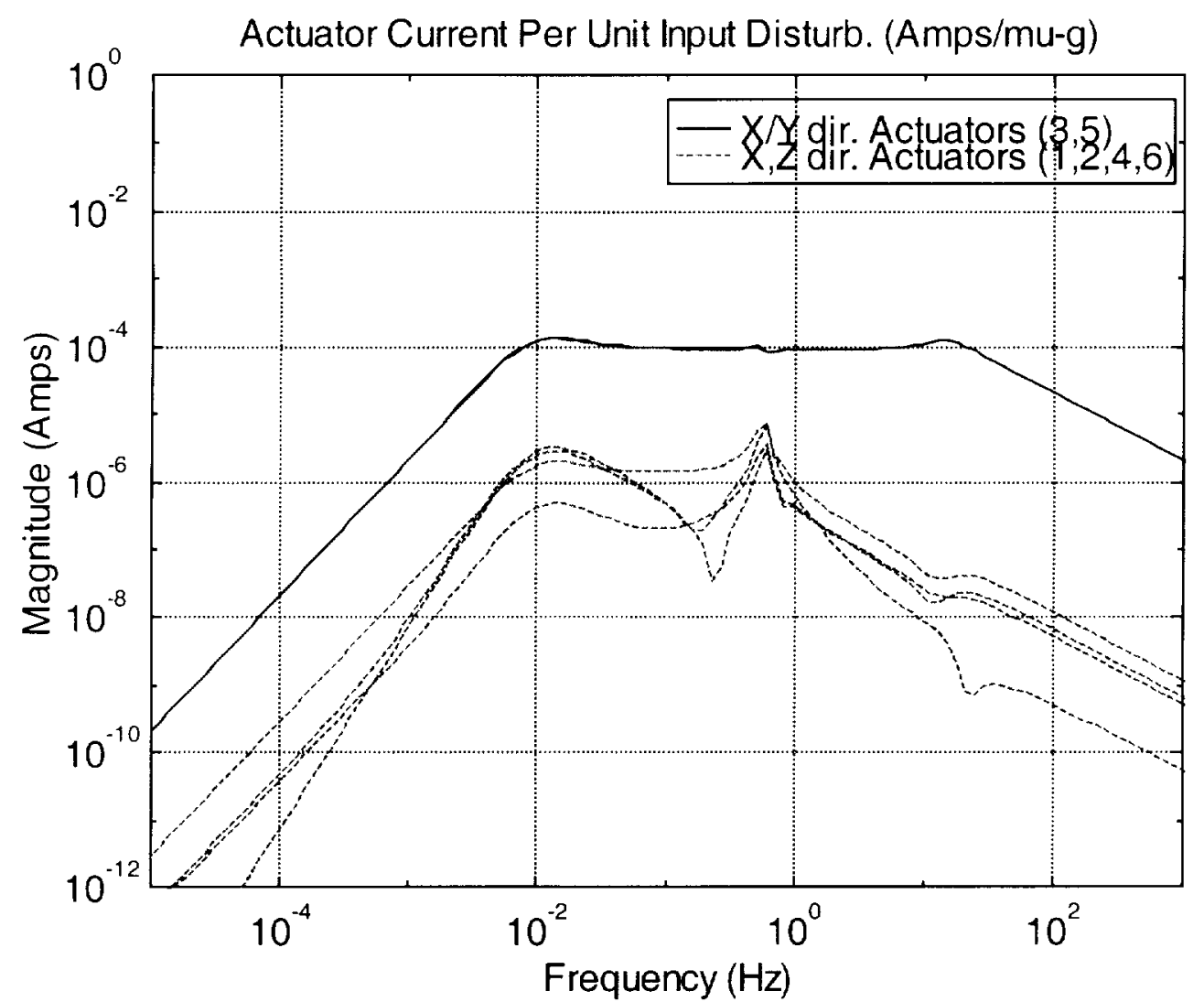

Figure 12 - Closed Loop Current Vs. Frequency Y-Axis Direct Acceleration Disturbances (H2 Controller) 


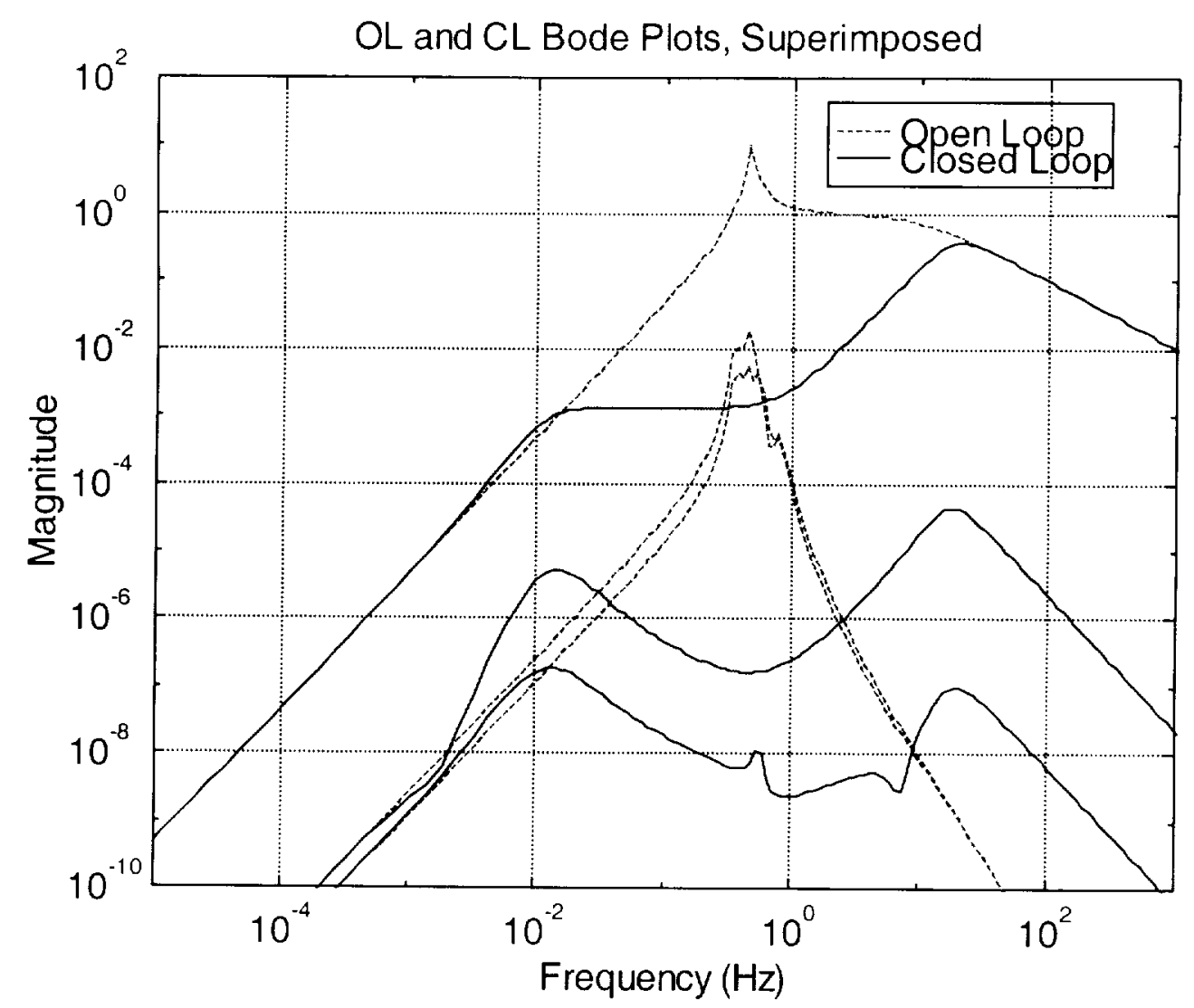

Figure 13 - Open and Closed Loop Transmissibilities for Z-Axis Direct Acceleration Disturbances (H2 Controller)

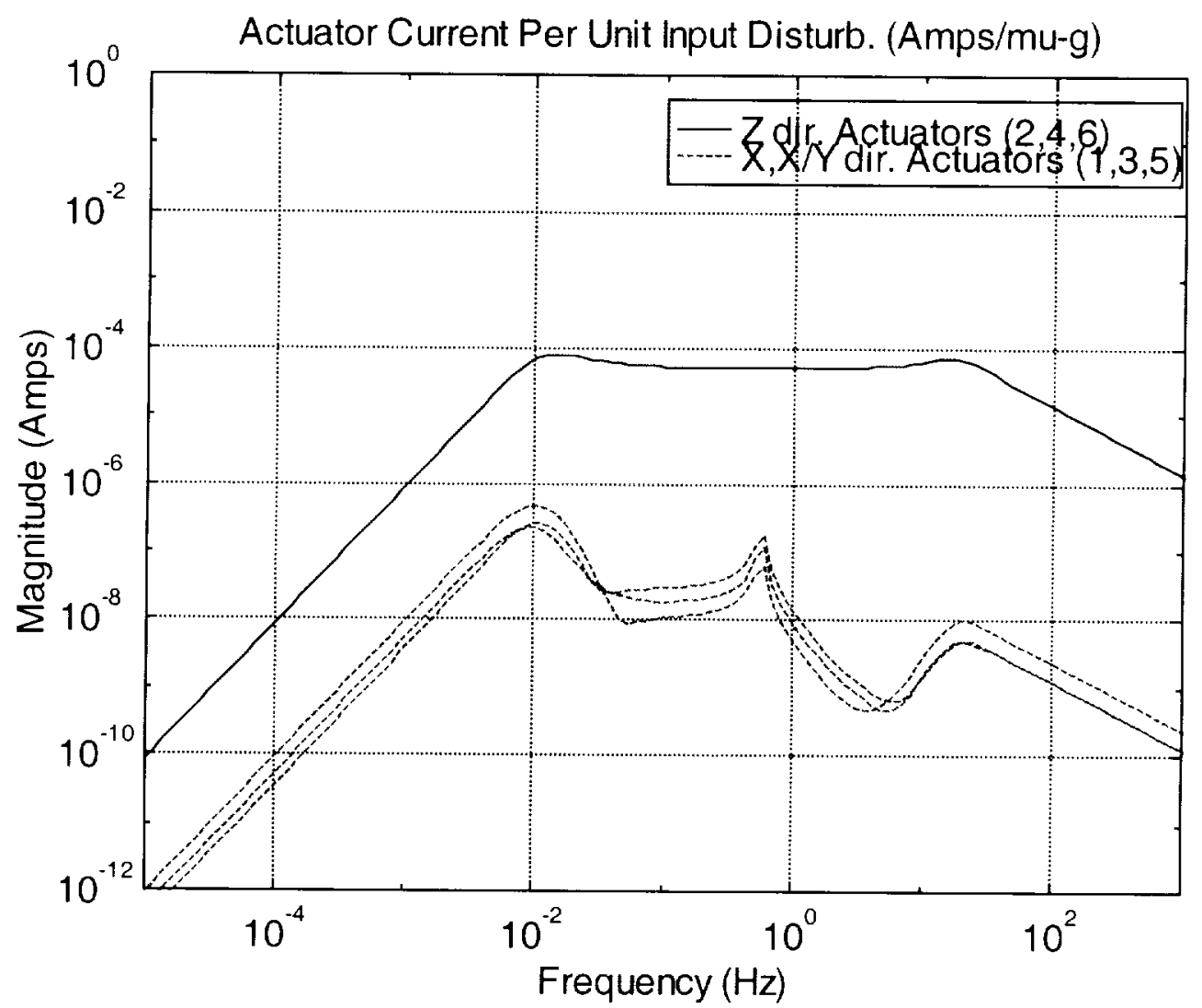

Figure 14 - Closed Loop Current Vs. Frequency Z-Axis Direct Acceleration Disturbances (H2 Controller) 


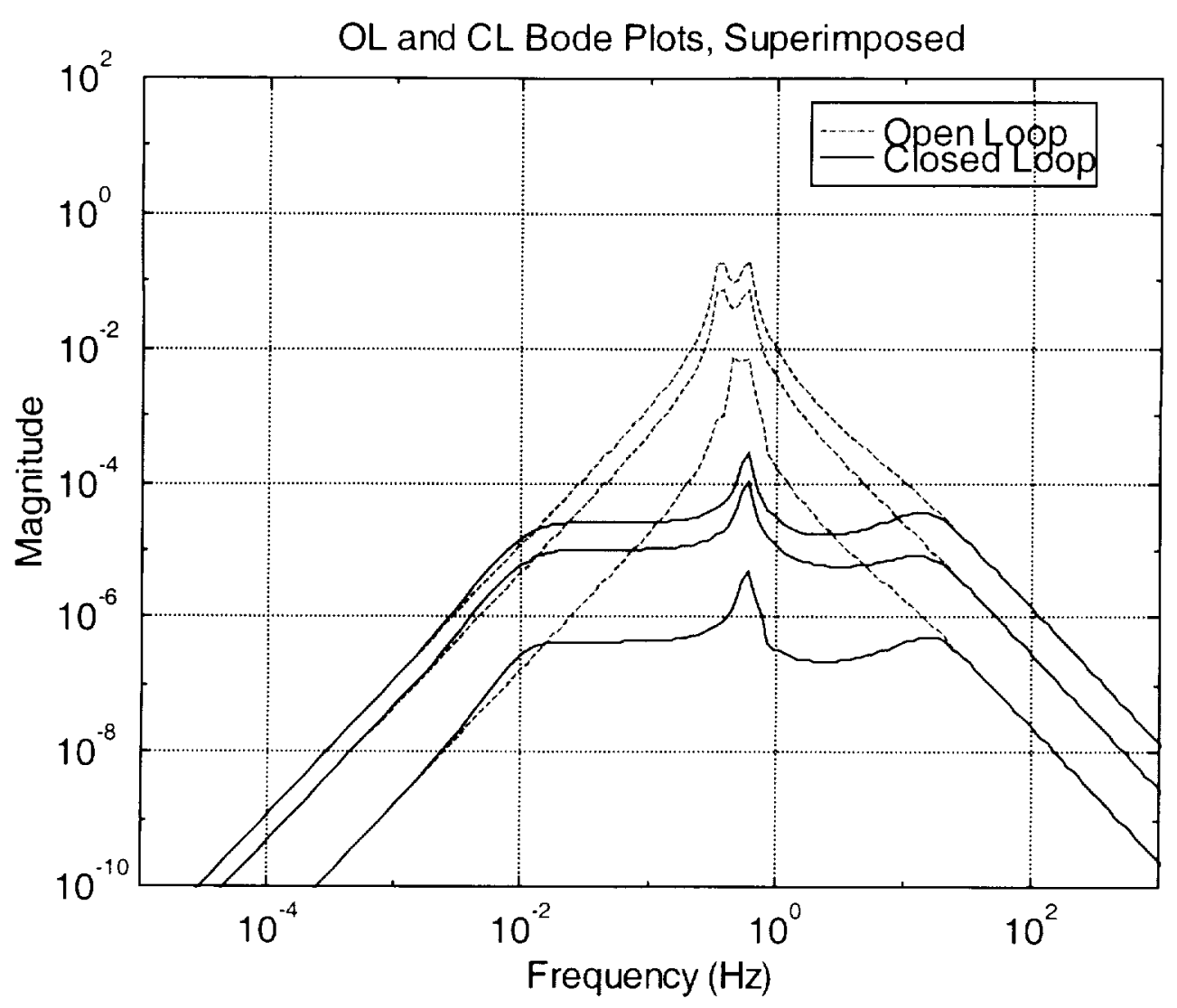

Figure 15 - Open and Closed Loop Transmissibilities for $\mathrm{X}$-Axis Direct Angular-Acceleration Disturbances (H2 Controller)

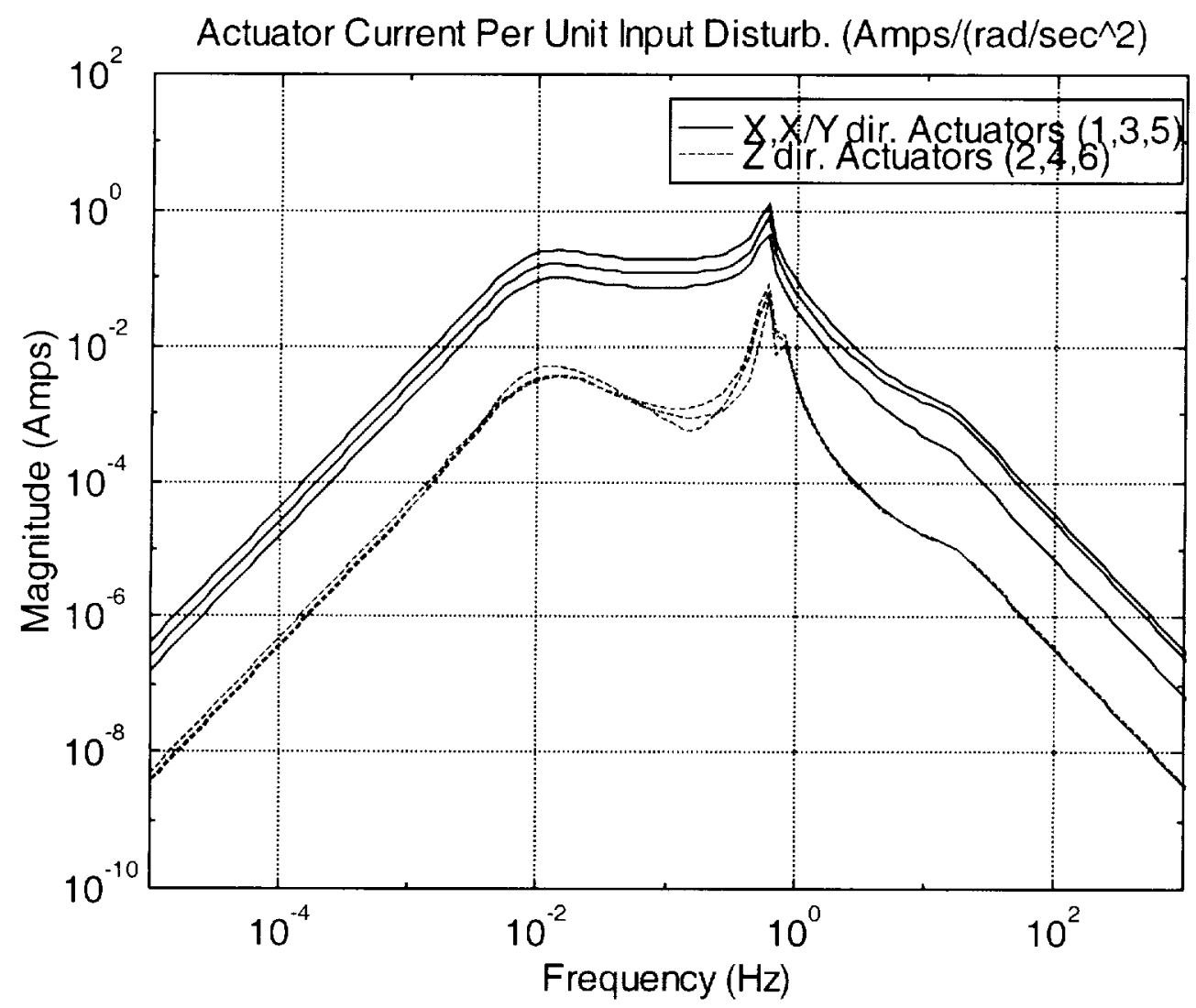

Figure 16 - Closed Loop Current Vs. Frequency $\mathrm{X}$-Axis Direct Angular-Acceleration Disturbances (H2 Controller) 


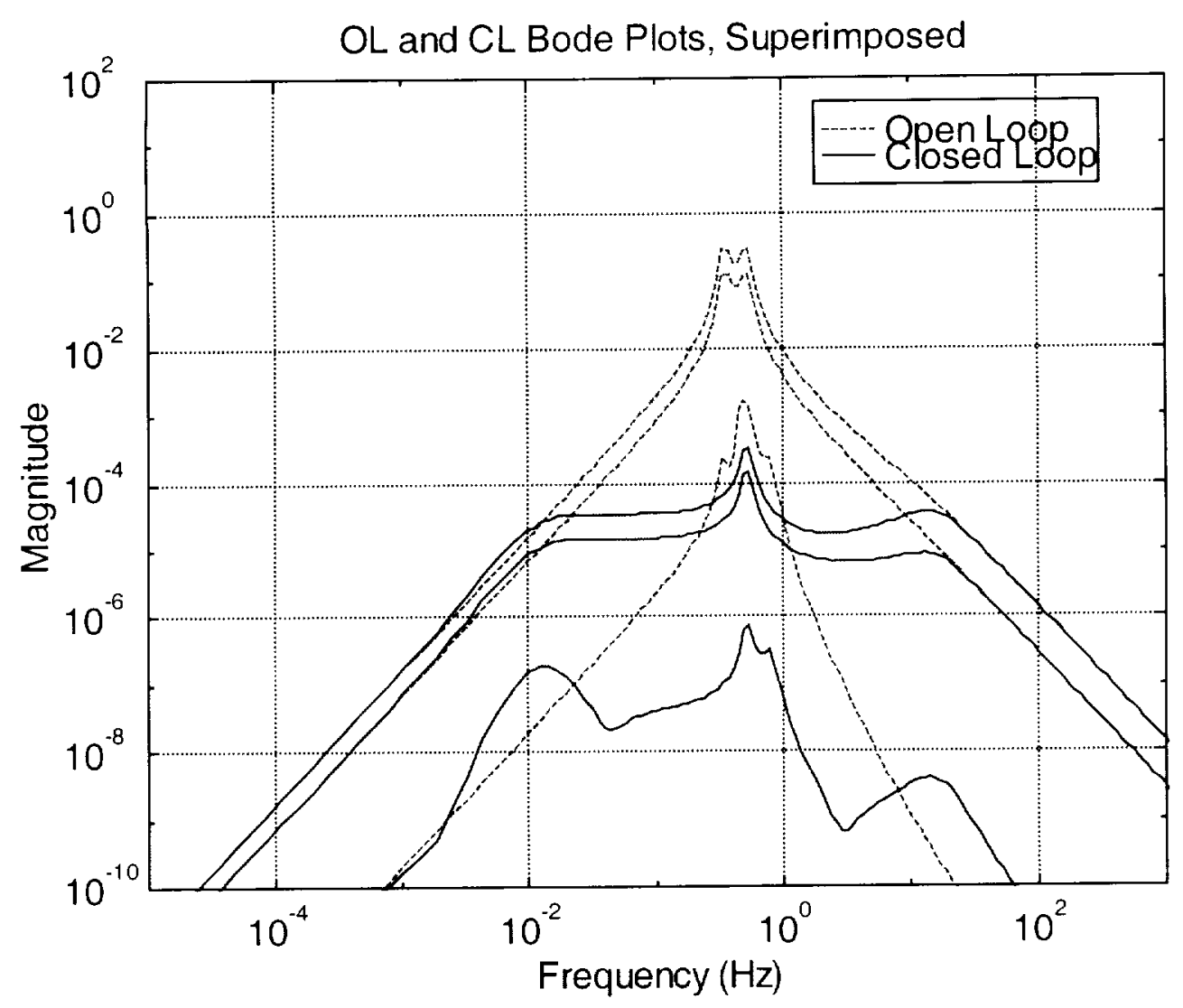

Figure 17 - Open and Closed Loop Transmissibilities for Y-Axis Direct Angular-Acceleration Disturbances (H2 Controller)

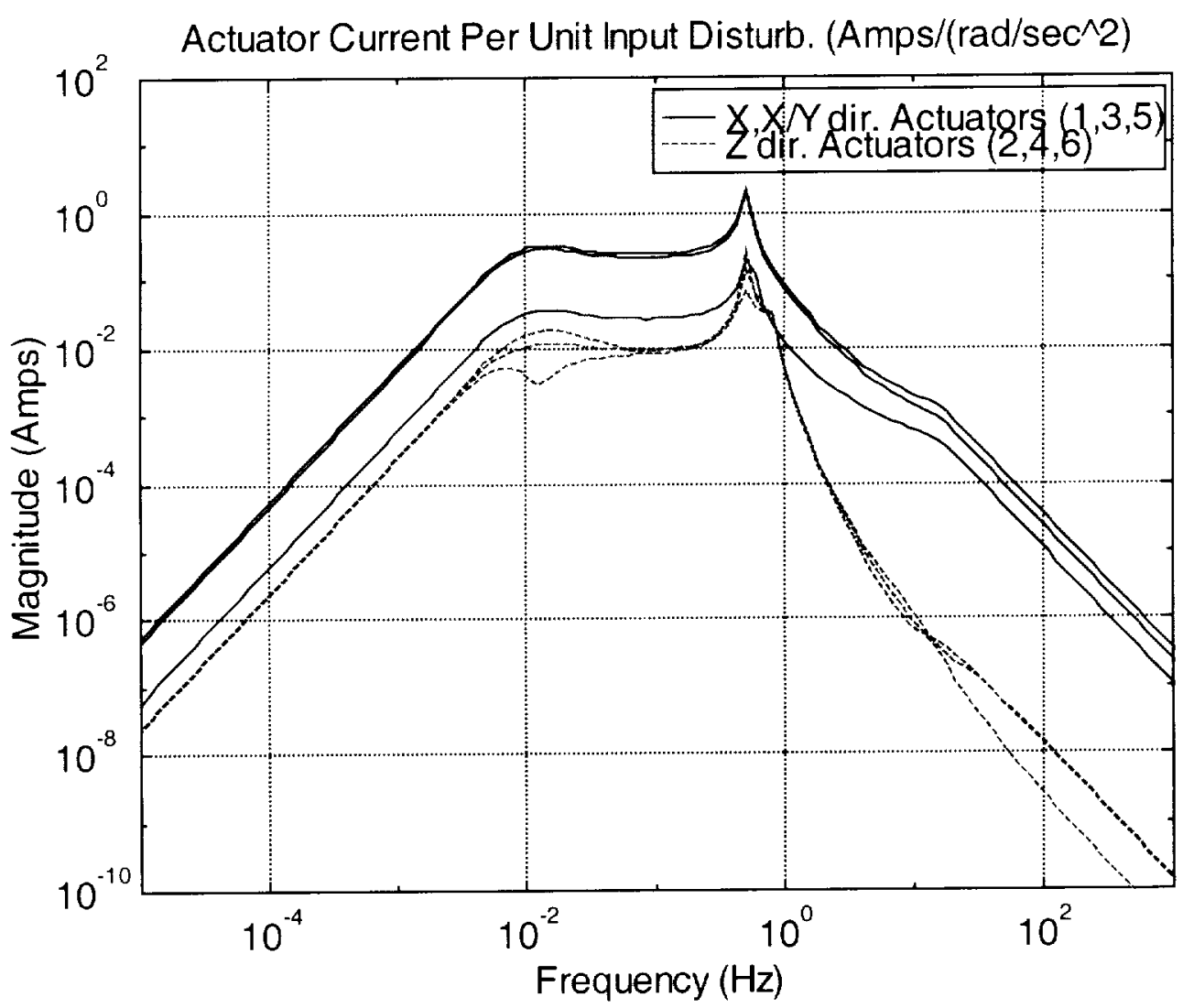

Figure 18 - Closed Loop Current Vs. Frequency Y-Axis Direct Angular-Acceleration Disturbances (H2 Controller) 


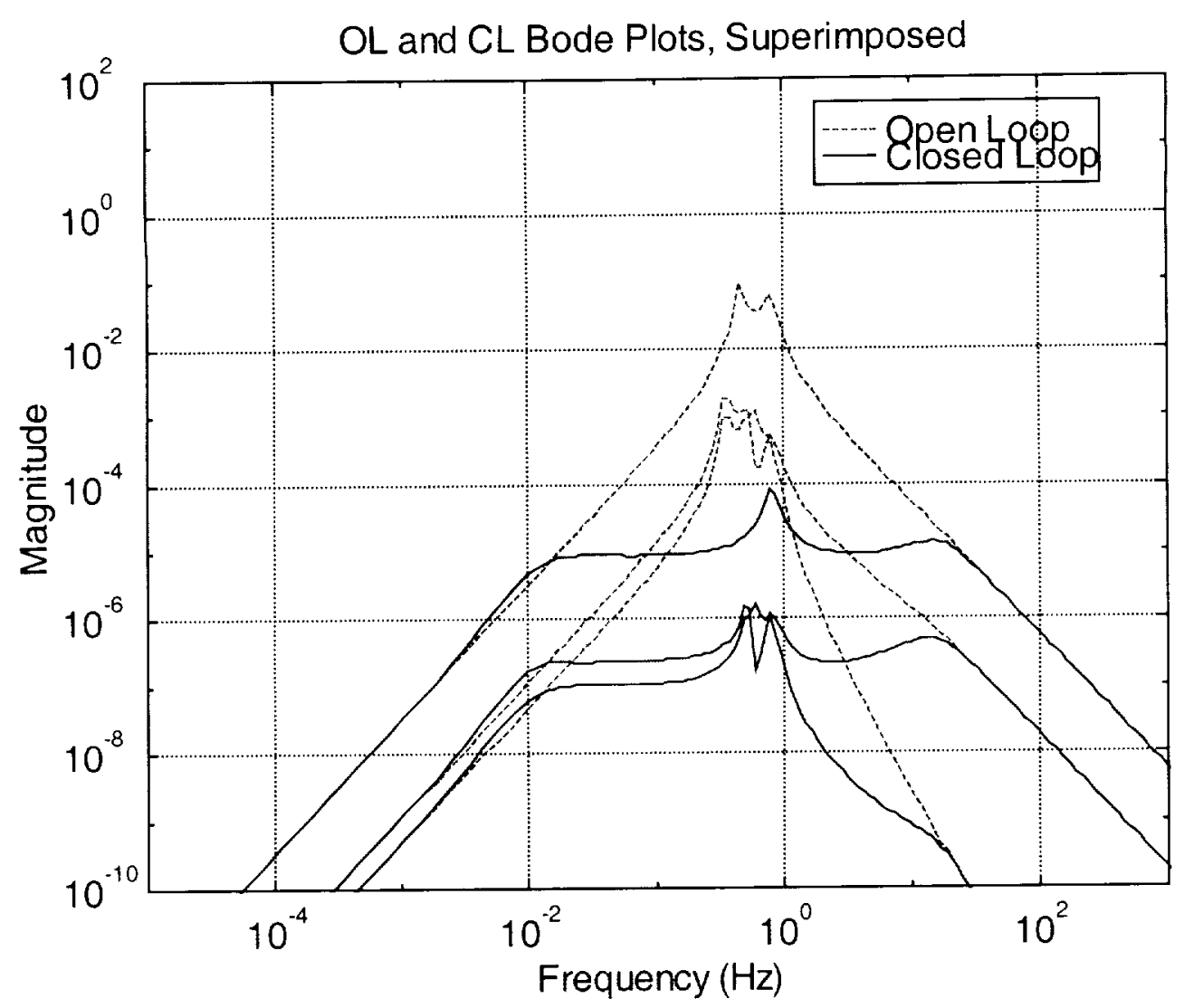

Figure 19 - Open and Closed Loop Transmissibilities for Z-Axis Direct Angular-Acceleration Disturbances (H2 Controller)

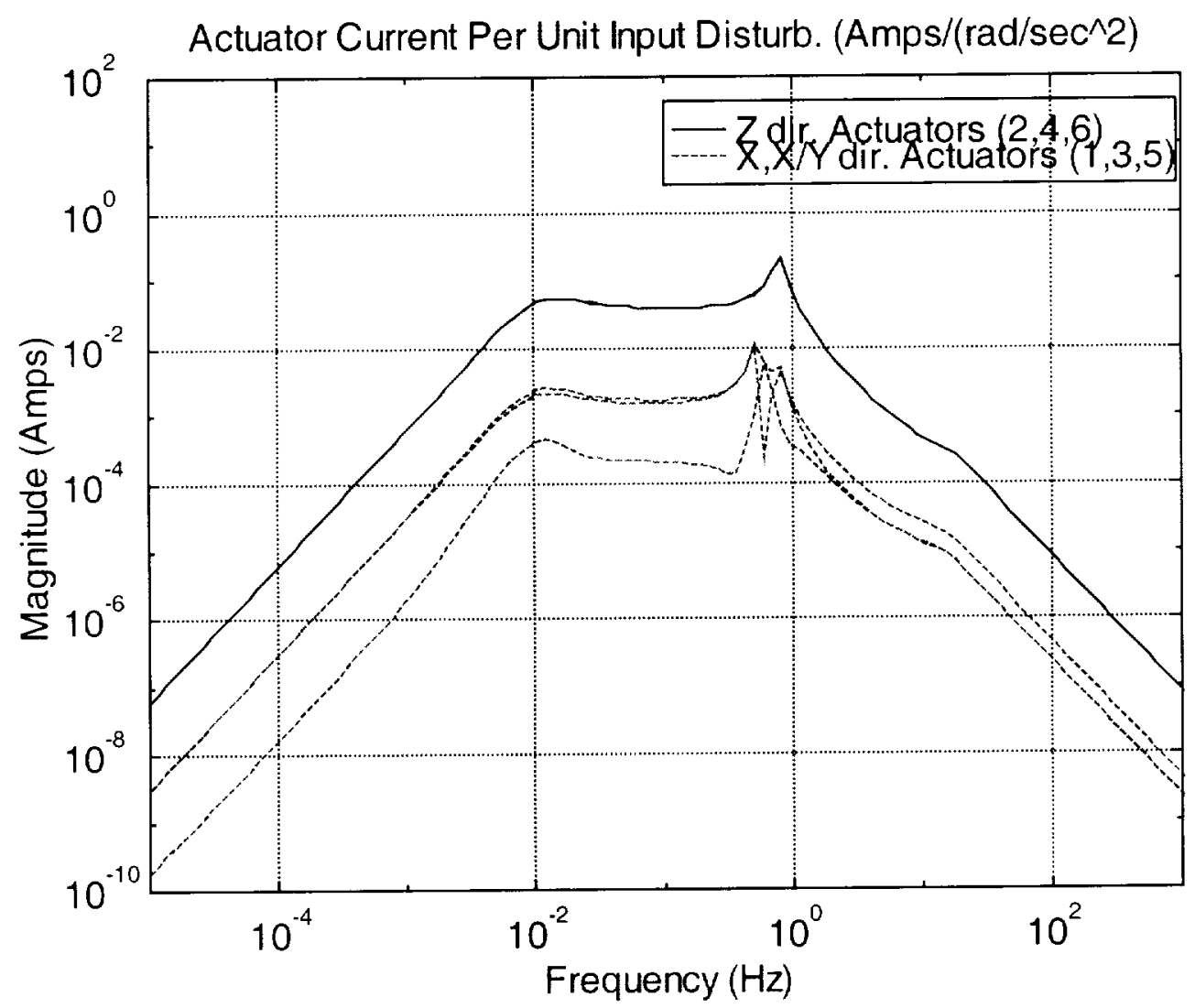

Figure 20-Closed Loop Current Vs. Frequency

Z-Axis Direct Angular-Acceleration Disturbances (H2 Controller) 


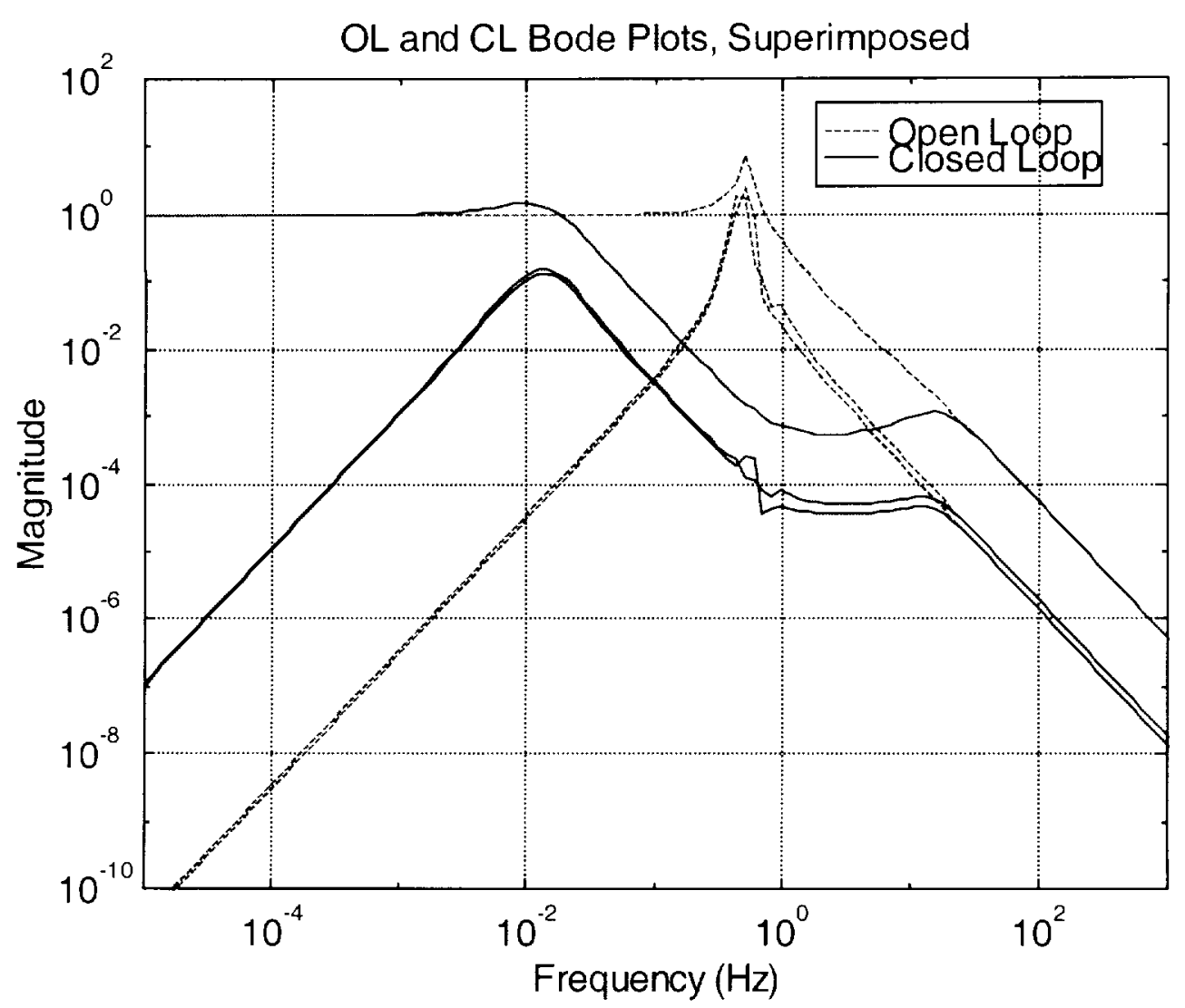

Figure 21 - Open and Closed Loop Transmissibilities for Z-Axis Indirect Acceleration Disturbances (H2 Controller - Robustness Test)

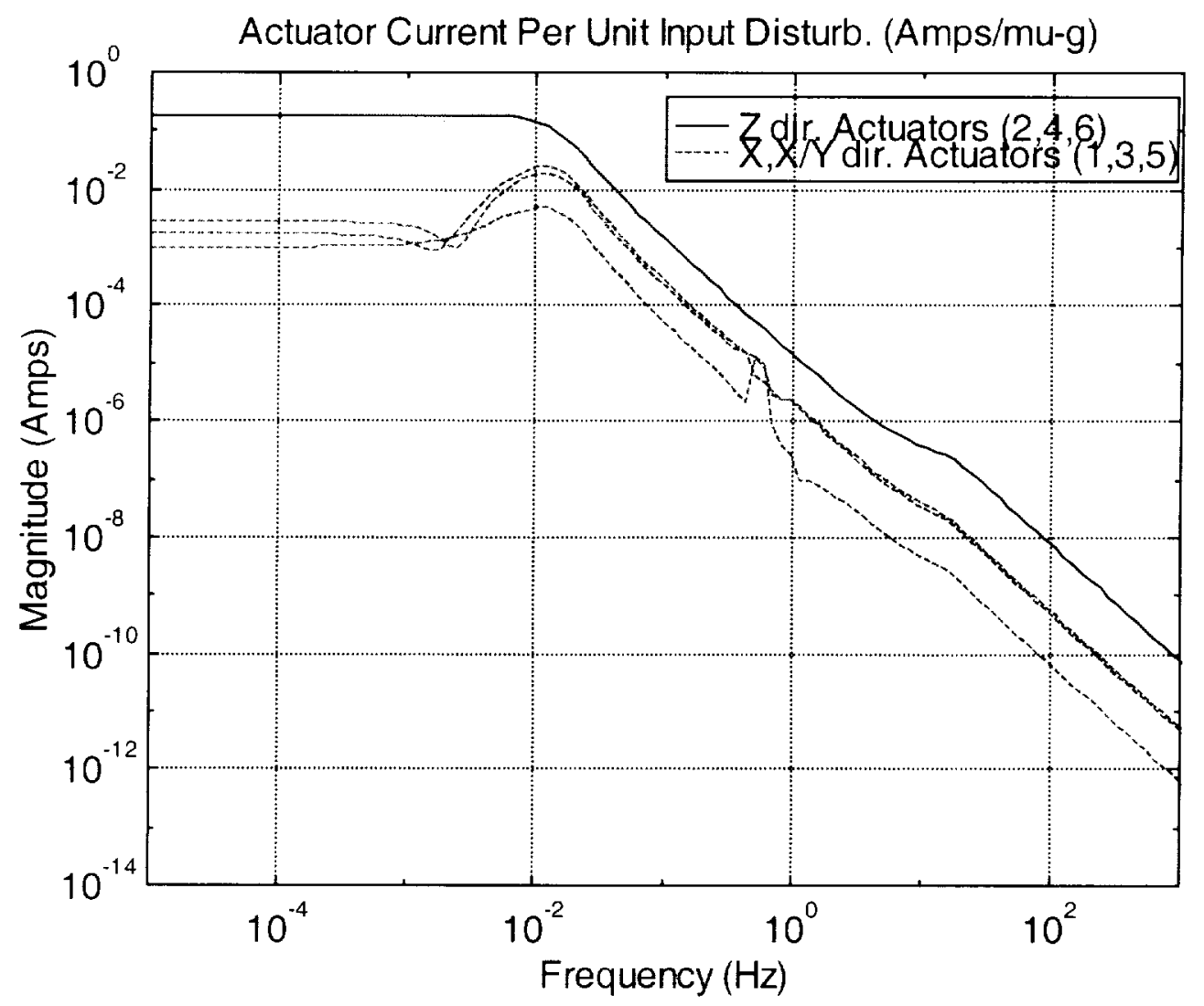

Figure 22 - Closed Loop Current Vs. Frequency Z-Axis Indirect Acceleration Disturbances (H2 Controller - Robustness Test) 


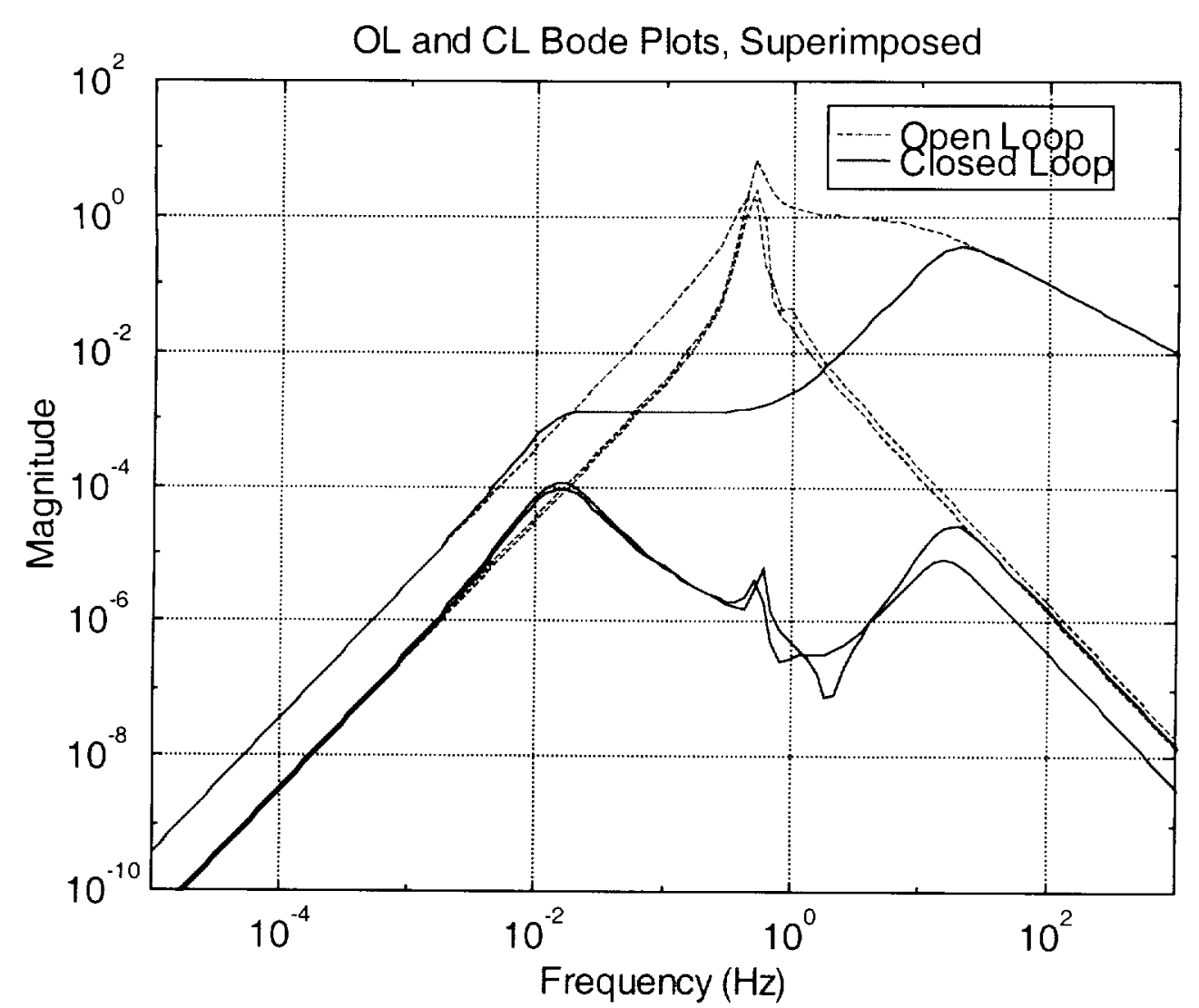

Figure 23 - Open and Closed Loop Transmissibilities for Z-Axis Direct Acceleration Disturbances (H2 Controller - Robustness Test)

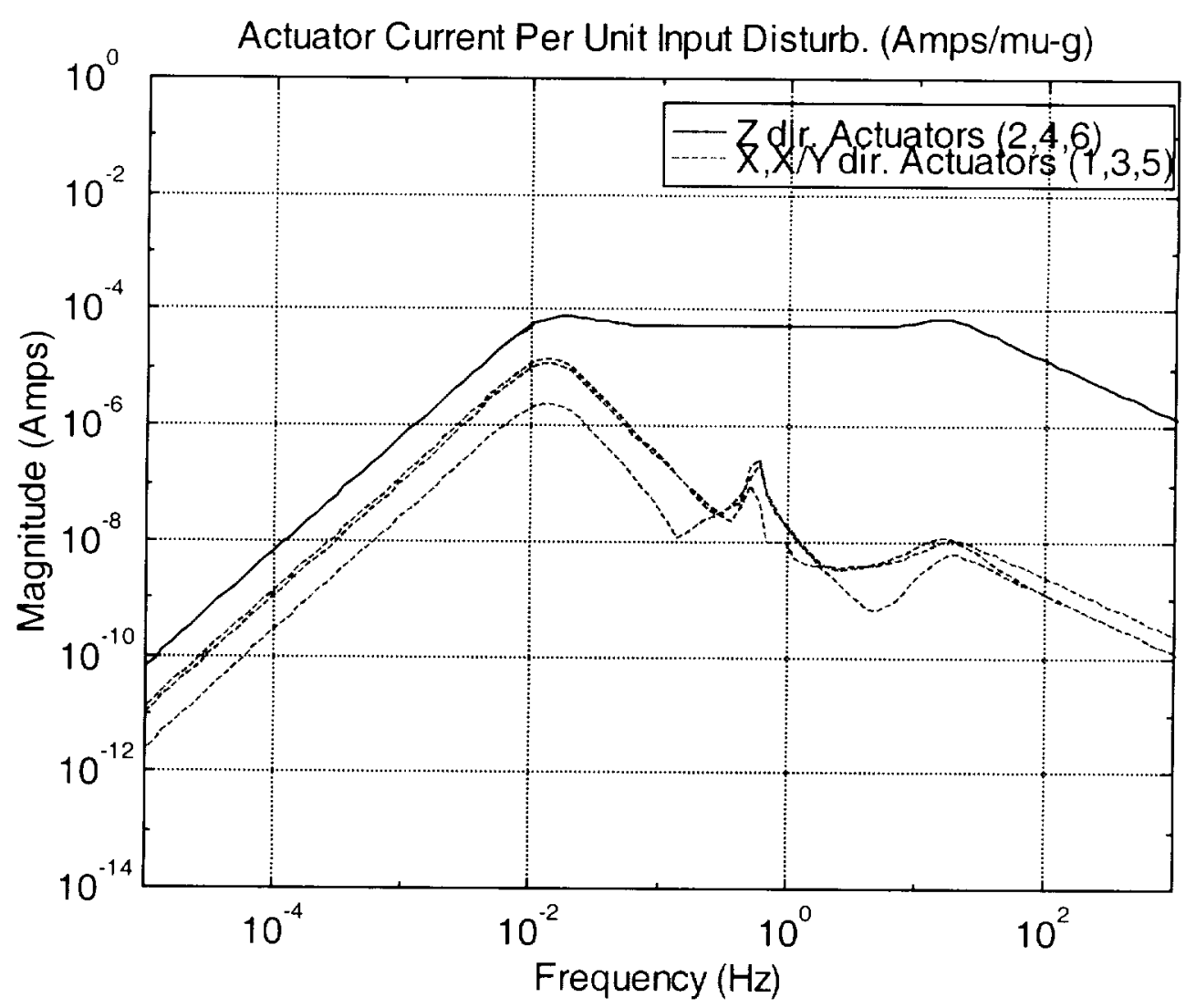

Figure 24 - Closed Loop Current Vs. Frequency Z-Axis Direct Acceleration Disturbances (H2 Controller - Robustness Test) 


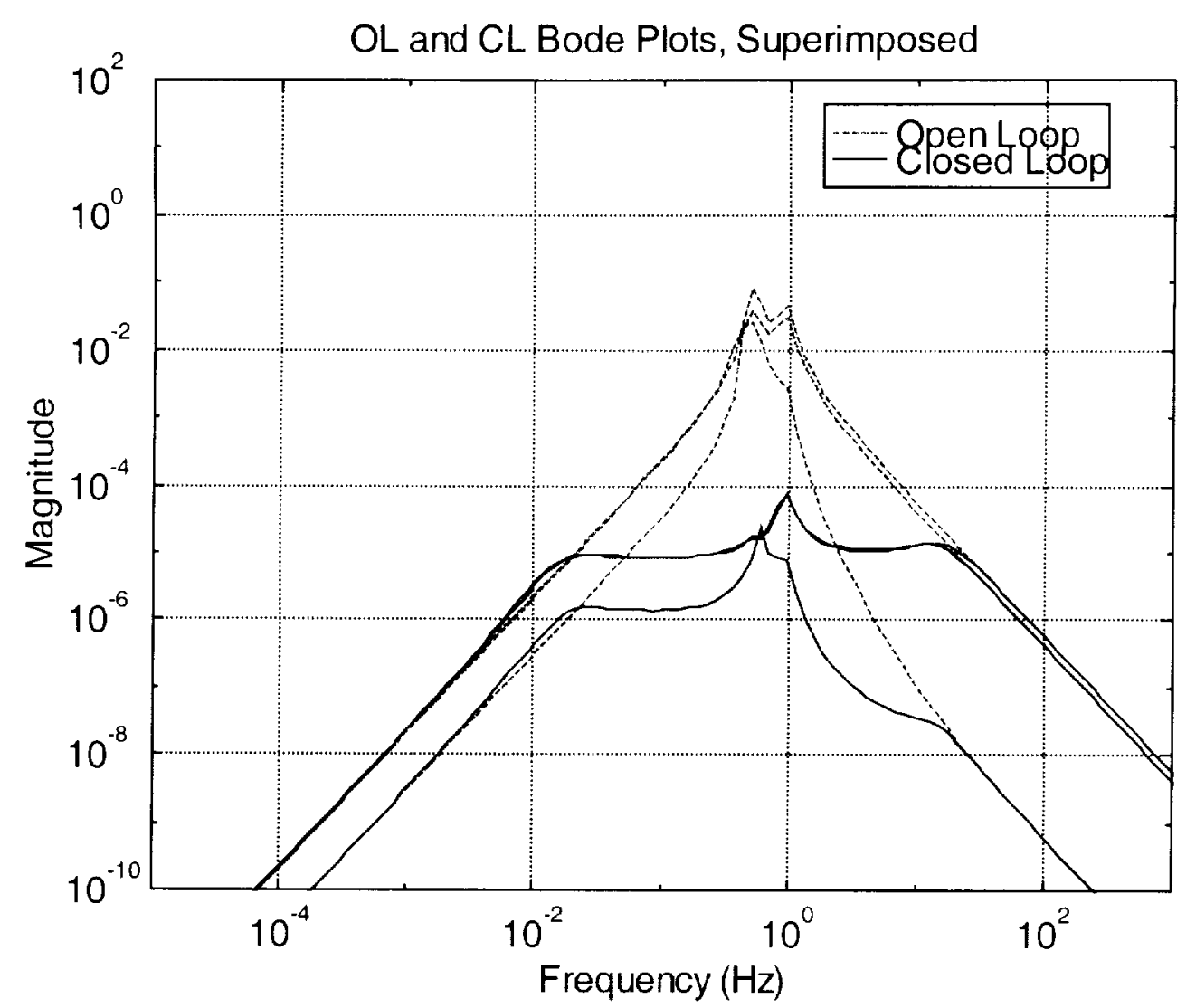

Figure 25 - Open and Closed Loop Transmissibilities for Z-Axis Direct Angular-Acceleration Disturbances (H2 Controller - Robustness Test)

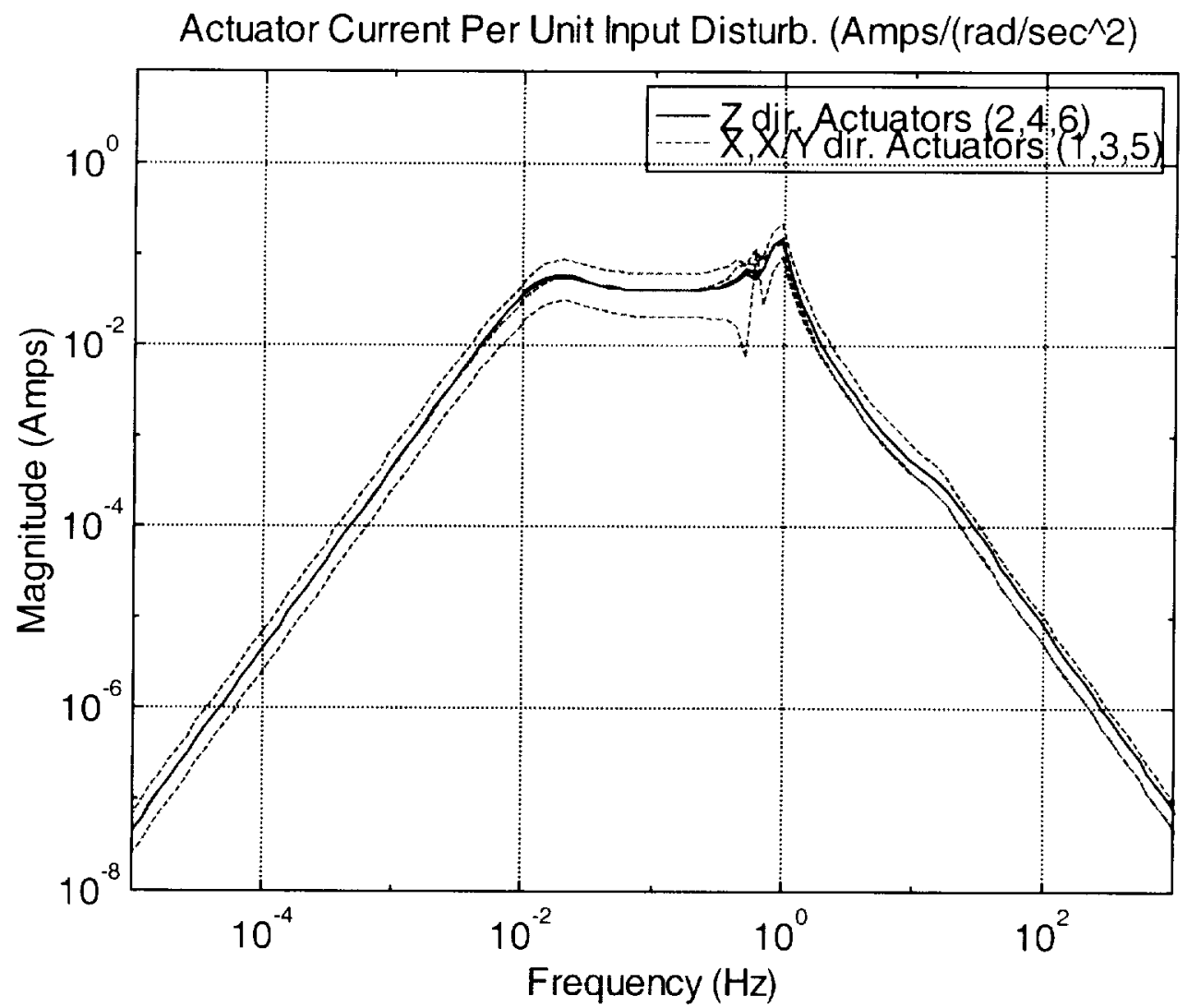

Figure 26 - Closed Loop Current Vs. Frequency

Z-Axis Direct Angular-Acceleration Disturbances (H2 Controller - Robustness Test) 